Université de Picardie Jules Verne

Faculté de Médecine d'Amiens

Année 2017

thèse $N^{\circ}$ 2017- 3

\title{
VALEUR DU RAPPORT NEUTROPHILES/LYMPHOCYTES POUR LE DIAGNOSTIC DES INFECTIONS BACTERIENNES.
}

\section{THÈSE D'ETAT DE DOCTEUR EN MEDECINE Mention Spécialité}

Présentée et soutenue publiquement le 20 janvier 2017

Par Madame Agathe LEGRAIN

Président du Jury :

Monsieur le Professeur Pierre DUHAUT

Juges :

Monsieur le Professeur Jean-Luc SCHMIT

Madame le Professeur Sandrine CASTELAIN

Monsieur le Professeur Julien MAIZEL

Directeur de thèse :

Monsieur le Docteur Jean SCHMIDT 


\title{
REMERCIEMENTS
}

\author{
À Monsieur le Professeur Pierre DUHAUT \\ Professeur des Universités-Praticien Hospitalier \\ (Médecine Interne)
}

Chef du service de Médecine interne et maladies systémiques

Pôle "Médico-chirurgical digestif, rénal, infectieux, médecine interne et endocrinologie"

(D.R.I.M.E)

Merci de votre enseignement.

Evoluer dans votre service a été un privilège.

Merci de me faire l'honneur de présider mon jury. 


\begin{abstract}
À Monsieur le Professeur Jean-Luc SCHMIT
Professeur des Universités-Praticien Hospitalier

(Maladies infectieuses et tropicales)

Responsable du service des maladies infectieuses et tropicales

Pôle "Médico-chirurgical digestif, rénal, infectieux, médecine interne et endocrinologie"

(D.R.I.M.E)
\end{abstract}

Chevalier dans l'Ordre des Palmes Académiques

Votre passion pour la médecine et votre humanité sont un exemple. Je suis fière de pouvoir continuer à apprendre et exercer dans votre service. Merci de me faire l'honneur de participer à ce jury. 


\section{À Madame le Professeur Sandrine CASTELAIN}

Professeur des Universités - Praticien Hospitalier

(Bactériologie, virologie-hygiène hospitalière)

Laboratoire de Bactériologie et Virologie

Pôle biologie, pharmacie et santé des populations

Merci de m'avoir ouvert les portes de votre laboratoire pour que je complète ma formation d'infectiologue.

Merci de me faire l'honneur de participer à ce jury. 


\section{À Monsieur le Professeur Julien MAIZEL \\ Professeur des Universités-Praticien Hospitalier}

(Réanimation, médecine d'urgence)

Découvrir la réanimation à tes côtés a été enrichissant tant sur le plan humain que des connaissances.

Ta pédagogie, ta rigueur et ta disponibilité ont contribué à rendre cette discipline passionnante à mes yeux.

Merci de me faire l'honneur de participer à ce jury. 
A Monsieur le Docteur Jean SCHMIDT

Maître de Conférences des Universités - Praticien Hospitalier

Médecine interne

J'ai beaucoup appris à tes côtés au cours de cet internat.

Ton calme et ta conscience professionnelle au cours des deux semestres passés en médecine interne m'ont aidé à progresser.

Merci d'avoir dirigé ce travail, de ta patience et de tes conseils. 
La présentation de cette thèse signe la fin de douze ans de travail, d'émotions, de doutes, de joies et surtout de rencontres qui contribuent à la construction de la personne et du médecin que je suis devenu.

C'est pour moi l'occasion de vous remercier. Je n'en serai pas là sans :

Les médecins qui m'ont accompagnée dans ma formation et ont renforcé l'envie de faire ce métier :

- En médecine interne : Le Professeur Ducroix pour avoir fait germer ma vocation d'interniste et pour nos échanges jardiniers, Docteur Smail pour son dynamisme communicatif, Valery pour son enthousiasme et sa générosité ;

- En néphrologie : Le Docteur Makdassi pour son amour infini de la néphrologie ;

- En hématologie : Le Professeur Marolleau pour son enthousiasme, Bruno Royer «Monsieur Gentil » pour son dévouement et sa franchise, Bérengère pour sa force et son humilité, Anne pour sa sensibilité ;

- En réanimation médicale : Loay pour son investissement et son envie de nous apprendre, Bertrand de Cagny pour l'importance du travail « bien fait » et du bon sens avant tout ;

- En rhumatologie : Franck pour les heures passées à décortiquer des rachis et sacroiliaques avec passion.

- En pathologies infectieuses : Nathalie Landgraf et le Dr Pik pour avoir suivi mes premiers pas d'interne, Youssef pour ses conseils au quotidien et son envie de partager les connaissances, Jean-Philippe pour les discussions passées et futures sur nos passions communes et l'envie de faire bouger les choses, Cédric pour son aide précieuse dans mon parcours ;

Mes co-internes, qui sont devenus au fil des ans bien plus que des collègues : Gaëlle, Fanny, Chloé, Xiaoli, Morgane, Coralie, Marie, Anne, Charlotte, Juliette, Xavier, Amandine, Mathieu, Alexis, Pierre-Yves, Chandra, Magalie, Ophélie, Léa, Caroline, Alice, Thibaut, Ines, Julien, Rémy et Guillaume pour les collaborations anciennes et futures.

Les équipes para médicales: Monsieur Degouy, Laure «t'es belle », Léonie, Karine, Stéphanie, Virginie, Charlotte, Manue, Laïla, Elise, Astrid, Martine, Céline, Claire, Lucile, Catherine, Eléonore, Camille, Lucie, Ela, Lydia, Gwen, Isabelle, Emilie, Pépette, Manue, Sylvie, Céline, Valérie, Sabine, Thomas, Willy, Adrien, Aymeric, Mathieu, Laure, Mélanie, Julie, Sam, Anne, Camille, Assita et ceux que j'oublie, pour donner un sens au mot « équipe »!

Les secrétaires Perrine, Brigitte, Catherine, Véro et Isabelle pour leur disponibilité et leur patience. 
Mes oncles et tantes, cousins et cousines pour avoir suivi de près ou de loin mon parcours et m'avoir toujours encouragé. Particulièrement Claire et Ying pour les ballades irlandaises, Marie pour Pascal, Adrien et Eneli pour l'accueil Versaillais, Pierre-Edouard et Alix pour les virées parisiennes, Lucie pour les fous rires sur le chemin de l'école. Mes filleules Rachel et Gladys pour les bons moments ;

Isabelle et Gaby, Manue et Seb, Sophie, Johan et Magalie pour les metkas, kluskis, cours de tricot et de couture à venir, parce qu'il n'y a pas que le boulot dans la vie ... ;

Les copains de l'externat, ceux que je ne vois plus beaucoup mais que je n'oublie pas : Sophie « mon poussin », Jade, Christophe, Caro, Rami, Nima, Max, Julien, Rita, Yassine, Hamza, Hamidour, Hassan, William, Laurent, Alexis, Christo...

Emilie, ma kiasse, pour les fous rires, délires, rendez-vous au coin et monaco au Kerry ;

Rosa pour la P1, ses repas, ses QCM, stress, virées lilloises et champêtres pleines de secrets, pour les micro-pauses et micro-siestes ;

Rémi mon fils et Emilie, Ben et Eugénie, Raphael Bonne-Santé et Aurélie, Xavier et Julie, Nico et Emilie pour me rappeler qu'il y a une belle vie en dehors des hôpitaux et des bouquins ;

Ceux qui de chefs sont passés au rang d'amis : Francois pour les pizzas en garde et les checklist, Marianne pour éclairer cette sombre discipline qu'est la dialyse, Pauline pour sa douceur et sa confiance, Florence pour sa bonne humeur ;

Clément G. pour son soutien et pour les secrets plus si secrets ;

Myriam pour le soleil qu'elle apporte à son contact ;

Audrey pour son sérieux un brin audacieux et tellement attachant ; 
Marie pour ces deux semestres, pour les échos de tophii et les pizzas maison plus ou moins partagées ;

Bébé Sarah pour les pauses-goûter, les échographies secrètes et les épis à deux ;

Clément Brault «Mister Sandman » pour les petites musiques, textos incongrus, pour ses cours sur les zombis et le Horton (aussi) ; Mathilde pour son sourire et son brin de folie qui ont contribué à rendre le semestre de réa aussi incroyable ;

Pauline «Paulette », ma collègue de congrès pour les craquages de fin de stage, les thés et la soucieuse relecture de ce papier ;

Maryam pour sa bonne humeur et son écoute attentive quand on en a besoin .

Maelle pour les fous rires à Creil et par la suite, pour être un peu chti au fond d'elle, pour le caramel quand même ;

Xavier pour les renards, pour les coups de folie et apéros improvisés ; Jess pour apporter un peu du Nord à notre quotidien picard, pour la coloc' et pour son soutien ... viril ;

Maité, pour son vrai management d'une drôle d'équipe d'internes, pour sa sensibilité et les visites inoubliables. Jérôme pour ta patience dans nos soirées un peu trop « médecins »;

Yoann pour les trucs en -ose, les clips, les pommes de terre, les imitations, pour le mec de chez Tung et les soirées pyjamas. Cécile pour son optimisme et ses conseils toujours précieux, pour les fraises et les petits pains à la cannelle ;

Dimitri «Philippe !» «Benjam'» pour les cathés, les sushis et les bagarres, pour avoir été un co-interne et un chef à la hauteur. Aurélie pour sa bonne humeur et son courage à nous écouter raconter les mêmes histoires ; 
Annis pour l'inté et ses apéros un peu fous, pour les soirées BU, les escapades à Rome, Ubud, Amsterdam et les photos, pour rester toi jusque dans les chemins de halage. Mathilde pour être à la hauteur de ce sacré bonhomme ;

Stéphane mon frère plus que confrère, pour être là depuis le syndrome cave supérieur jusqu'à aujourd'hui, des repas MTV aux soirées top-chef, des sous colles au craquage post ECN, pour les Batel's et la réa Calmette, pour ton humanité qui font de toi un ami et médecin en or. Aurélie pour ton appui pendant toutes ces années, tes petits plats et attentions, pour les larmes de joie plutôt récurrentes et pour les futures escapades à 7 ;

Louison, Octave, Quentin, Cléa, Rose, Auguste, Aline, Romane, Marc, Bastien, Baptiste, Romain, Luna, Enzo, Lola, Zoé, Samuel, Gustave, Maxime, Thomas, Nelson, Harvey pour nous rappeler ce qui compte vraiment. 
Papy et Mamie pour leur amour et leurs pensées.

Mamie pour être si fière de nous. J'aurais aimé que tu sois là aujourd'hui.

Et puis il y a ceux pour qui les mots ne suffisent pas:

Ma famille, pour rester toujours solide et unie, pour passer les épreuves et les bons moments ensemble. Pour m'avoir soutenue dans mes angoisses, mes doutes et malgré mes humeurs. Pour avoir fait ce que je suis aujourd'hui ;

Mathilde mon chou-chéri, ma meilleure pour son écoute et sa patience à mon contact pas toujours facile ;

Antoine pour son flegme en toute circonstance et ses attentions discrètes mais toujours justes ;

Mon père pour m'avoir donné le goût de la science, pour son soutien à toute épreuve et sa fierté ;

Ma mère pour m'avoir fait comprendre qu'on ne se construit qu'au contact des autres et pour son incroyable humanité ;

Julien, pour m'aider à me dépasser tous les jours.

Pour ton amour, ta patience et ton écoute,

Pour continuer à me faire rire même si je suis parfois la seule,

Pour ton exigence et ton refus d'être « comme tout le monde»,

Pour être un père incroyable pour Zacharie,

Pour être toi et m'avoir aidé à devenir moi. 
À Zacharie, 
RESUME :

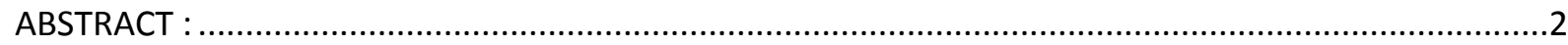

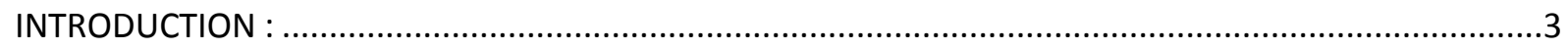

I. Contexte :

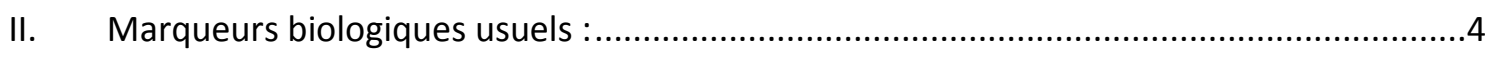

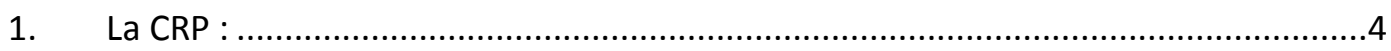

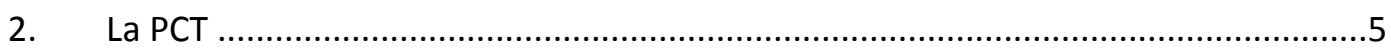

III. Le rapport polynucléaires neutrophiles/lymphocytes (RNL) .........................................

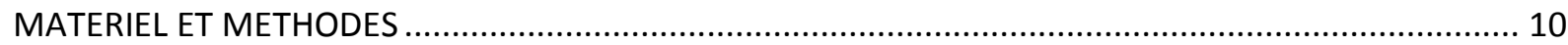

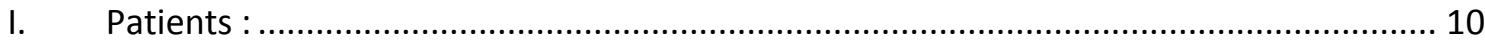

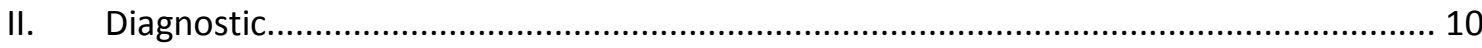

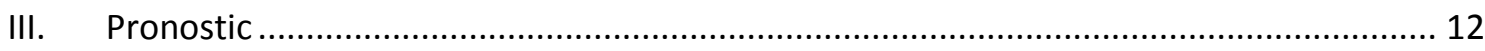

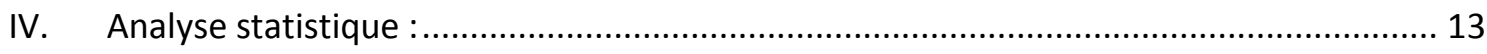

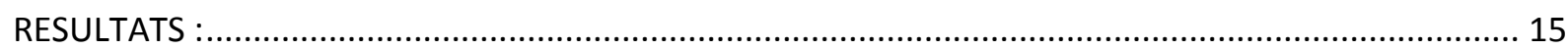

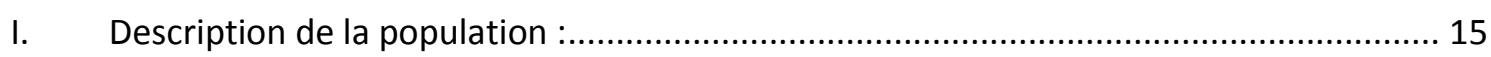

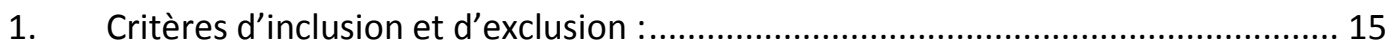

2. Diagnostics de sortie :.................................................................................... 16

3. Description des étiologies non bactériennes : .................................................... 18

4. Infections bactériennes : ............................................................................... 18

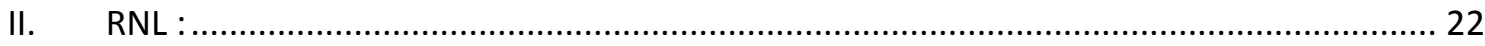

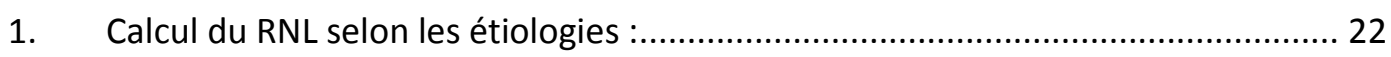

2. Performances diagnostiques des marqueurs usuels :....................................... 24

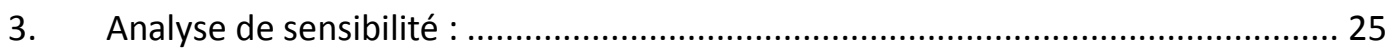

4. Performances du RNL pour le diagnostic de bactériémie.................................. 26 
III. Cas des patients âgés de plus de 75 ans :

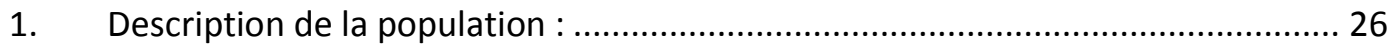

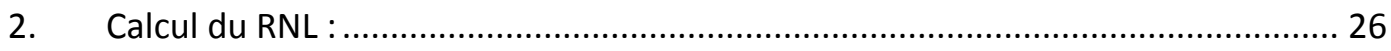

3. Comparaison des performances à celles de la CRP : ......................................... 28

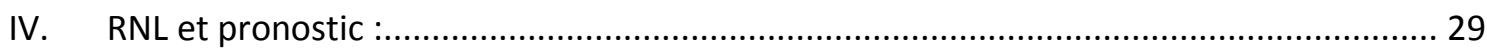

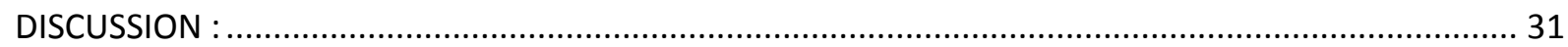

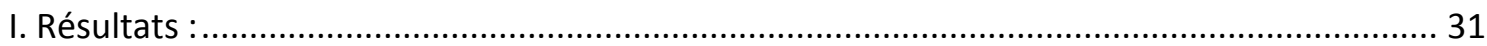

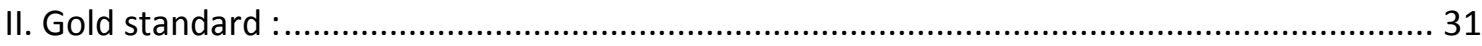

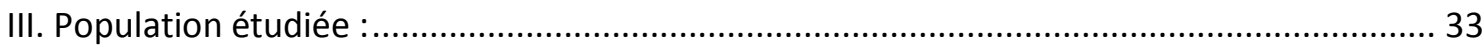

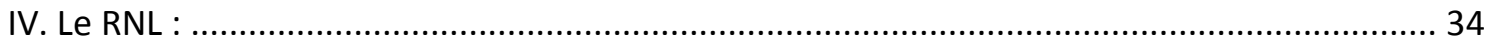

1. Intérêt pratique au quotidien :.......................................................... 34

2. Modalités d'utilisation du RNL ............................................................ 34

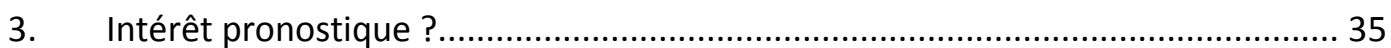

4. Autres utilisations du RNL :.............................................................. 36

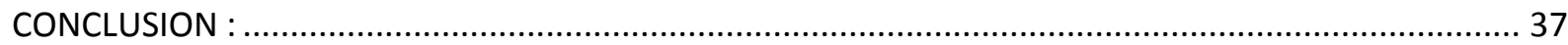

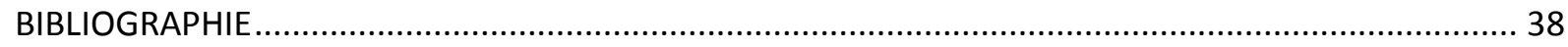




\section{RESUME :}

Introduction : En réaction à une infection bactérienne, les polynucléaires neutrophiles (PNN) circulants augmentent tandis que les lymphocytes diminuent par redistribution et majoration de leur apoptose. L'objectif était d'évaluer la valeur diagnostique du Rapport Neutrophiles sur Lymphocytes (RNL) pour différencier une étiologie bactérienne d'une autre cause devant une fièvre et/ou un syndrome inflammatoire chez un patient hospitalisé dans un service de médecine interne conventionnel.

Matériels et méthodes : Nous avons réalisé une étude rétrospective observationnelle sur les patients hospitalisés en 2014 pour une fièvre et/ou un syndrome inflammatoire. Leurs données cliniques, biologiques et examens microbiologiques et radiologiques étaient recueillis. Ils étaient classés en fonction du diagnostic final établi (bactérien, viral, parasitaire, thrombotique, néoplasique, inflammatoire ou autre). La valeur diagnostique du RNL pour les infections bactériennes était comparée à celle de la CRP, de la PCT et au nombre de PNN.

Résultats : 339 patients ont été inclus. Une infection bactérienne était diagnostiquée dans 38 $\%$ des cas. Le RNL médian était de 9,59 [5,47-16,70] chez les patients présentant une infection bactérienne, versus 5,38 [3,40-8,70], p < 0,0001, chez les autres. Au seuil optimal de 8,2, la sensibilité du RNL pour le diagnostic d'infection bactérienne était de $61 \%$ [IC $95 \%$ (52-69)], la spécificité de $73 \%$ [IC $95 \%$ (66-81)]. L'aire sous la courbe ROC était de 0,69 [IC $95 \%$ $(0,63-0,76)]$, sans différence significative avec l'aire sous la courbe ROC de la CRP ou de la PCT.

Conclusion : Un RNL élevé à l'admission constitue un argument pour une origine bactérienne à une fièvre et/ou un syndrome inflammatoire. Ces valeurs doivent être confirmées sur une cohorte prospective.

Mots clés : Rapport Neutrophiles sur Lymphocytes ; Infection bactérienne ; PCT ; Diagnostic 


\section{ABSTRACT :}

Introduction: In response to bacterial infection, circulating neutrophil count increase while lymphocytes decrease by redistribution and increase of apoptosis. The objective was to evaluate the diagnostic value of the Neutrophil-Lymphocyte count Ratio (NLR) to differentiate a bacterial etiology from another cause of fever and /or inflammatory syndrome in patient hospitalized in a conventional medicine department.

Material and Methods: We conducted an observational retrospective study on patients hospitalized for fever and /or inflammatory syndrome in 2014. Their clinical, biological, microbiological and radiological data were collected. They were classified according to the final diagnosis (bacterial, viral, parasitic, thrombotic, neoplastic, inflammatory or other). The diagnostic value of NLR for bacterial infections was compared to CRP, PCT and Neutrophil Count.

Résults: 339 patients were included. A bacterial infection was diagnosed in $38 \%$ of cases. Median NLR was 9.59 [5.47-16.70] for patients with bacterial infection, versus 5.38 [3.408.70], $\mathrm{p}<0.0001$, for the others. In using a cut off of 8,2, the sensitivity of NLR for the diagnosis of bacterial infection was $61 \%$ [95 \% CI (52-69)], and the specificity was $73 \%$ [95\% CI (6681)]. The area under the ROC curve was 0.69 [95 \% CI (0.63-0.76)], with no significant difference with CRP and PCT.

Conclusion: In our setting, high NLR at admission was associated with a bacterial origin to fever and /or inflammatory syndrome. These results have to be confirmed in a prospective cohort.

Keywords: Neutrophil-Lymphocyte Ration (NLR), Bacterial Infections, PCT, Diagnoses 


\section{INTRODUCTION :}

\section{Contexte :}

La suspicion d'infection devant une fièvre et/ou un syndrome inflammatoire est un motif fréquent d'hospitalisation dans les services de médecine interne.

Les causes mises en évidence sont variées et peuvent être regroupées en causes infectieuses (bactériennes, virales ou parasitaires), et non infectieuses (thrombotiques, néoplasiques, ou inflammatoires). Les présentations cliniques de ces différentes entités peuvent être similaires et le diagnostic rapide d'une infection bactérienne à l'admission d'un patient en service de médecine est parfois délicat.

Néanmoins, il est important de pouvoir identifier ces pathologies rapidement afin de pouvoir mettre en place un traitement adapté consistant en une antibiothérapie probabiliste ou documentée la plupart du temps.

Les objectifs sont ainsi de réduire la morbi-mortalité, la durée d'hospitalisation, l'utilisation des antibiotiques afin de réduire leur impact toxique et allergique, et écologique en lien avec une surconsommation ou une consommation inadaptée.

Différents critères sont proposés pour aider le clinicien à établir ces diagnostics. Ils sont composés d'arguments cliniques, biologiques et paracliniques.

Le gold standard du diagnostic d'infection bactérienne est l'identification du germe en cause, que ce soit de façon directe (isolement sur les prélèvements effectués sur le site de l'infection ou au niveau sanguin par hémocultures lors des phases bactériémiques) ou indirectes (antigénuries, sérologies...).

Celui-ci est néanmoins difficilement obtenu en routine et peut être soumis à des contraintes de réalisation (prélèvements profonds ou invasifs) et à des défauts de performances diagnostiques (sensibilité, spécificité). Ainsi, différentes études ont montré que les hémocultures ne mettent en évidence l'agent bactérien que dans un tiers des cas lors des sepsis ${ }^{(1)}$. 
Le délai d'obtention du résultat des hémocultures et de ces prélèvements microbiologiques est long, lié à un temps de culture prolongé (48 à 72h le plus souvent) alors que le diagnostic doit être fait le plus précocement possible.

Les critères biologiques utilisés pour l'orientation du diagnostic sont le plus souvent la CRéactive Protein (CRP), la Numération Formule Sanguine (NFS) notamment le nombre de leucocytes et neutrophiles avec, dans le cas des infections bactériennes, une hyperleucocytose à polynucléaires neutrophiles, et la Procalcitonine (PCT).

\section{Marqueurs biologiques usuels :}

\section{La CRP :}

La CRP est un marqueur sécrété essentiellement par le foie. A l'état physiologique, son taux sérique est inférieur à $1 \mathrm{mg} / \mathrm{L}$, avec des variations jusque $20 \mathrm{mg} / \mathrm{L}$. Sa production est augmentée sous l'influence de cytokines pro-inflammatoires dans les 6 heures suivant une infection et/ou un dommage tissulaire et suit ensuite une décroissance exponentielle. Ainsi, la synthèse hépatique est principalement stimulée par l'IL6, potentialisée par 1'IL7, IL1 et TNF $\alpha^{(2)}$.

La CRP joue un rôle propre dans le mécanisme inflammatoire en se liant aux cellules de l'inflammation notamment phagocytaires et aux bactéries et en activant et régulant le complément. Elle stimule également la réponse immunitaire en favorisant la production d'IL8 par les polynucléaires neutrophiles et ainsi leur adhésion aux cellules endothéliales. Elle est donc impliquée dans la dégradation antigénique des infections et dans la régulation de la destruction des cellules endommagées, jouant un rôle dans la réparation et la cicatrisation.

Ainsi, l'augmentation de son taux sérique est peu spécifique, puisqu'elle intervient lors de dommages tissulaires, pouvant être causés par un processus tumoral, inflammatoire, thrombotique ou infectieux ${ }^{(3)}$. Elle est toutefois utilisée de façon courante en pratique, fournissant des éléments d'orientation devant un tableau clinique atypique ou en vue du suivi d'une pathologie infectieuse ou inflammatoire.

Une revue de la littérature de Simon et al réalisée en 2004 étudiait les valeurs diagnostiques des 
marqueurs usuels pour le diagnostic des infections bactériennes par rapport aux autres causes d'élévation de ce marqueur. Pour la CRP, la sensibilité était de $75 \%$ [IC 95 \% (62-84 \%)], et la spécificité de $67 \%$ [IC 95\% (56-77\%)] ${ }^{(4)}$.

\section{La PCT}

Le dosage de la PCT a été étudié depuis le début des années 90 concernant le diagnostic d'infection bactérienne ${ }^{(5)}$.

Ce marqueur est un précurseur de la calcitonine, sécrété à l'état physiologique par les cellules neuroendocrines de la glande thyroïde et du poumon, puis rapidement clivé pour la production de calcitonine, utilisée pour maintenir l'homéostasie calcique. Son taux est variable mais ne dépasse pas $0,5 \mathrm{ng} / \mathrm{mL}$ à l'état physiologique.

Il a été montré que dans les états d'infection, en particulier systémiques (sepsis), l'expression du gène codant pour la PCT (CALC-1) est stimulée notamment par l'IL1 et dans une moindre mesure par l'IL6 et le TNF $\alpha$. Cela aboutit à une synthèse par les différents organes et stimulant la réponse inflammatoire. Elle est inhibée par l'interféron $\gamma$, produit lors des infections virales ${ }^{(1)}$.

Ceci permet de penser que le dosage de ce marqueur pourrait être efficace pour diagnostiquer un état infectieux bactérien devant une fièvre ou un syndrome inflammatoire. Néanmoins, les différentes études réalisées montrent des résultats contradictoires, avec des valeurs plus intéressantes pour le diagnostic de sepsis chez les patients en état critique que pour les infections localisées ${ }^{(1,3)}$. Pour cela, et en raison d'un coût plus important, son utilisation est plus limitée en pratique quotidienne et elle est plus rarement dosée dès l'admission d'un patient.

La même revue de la littérature de Simon et al. évaluait la sensibilité de la PCT pour les infections bactériennes à $88 \%$ [IC $95 \%(62-84 \%$ )] et la spécificité à $81 \%$ [IC $95 \%$ (67-90 $\%$ )], pour tous types d'infections bactériennes confondues ${ }^{(4)}$. La méta-analyse de Wacker $e t$ al. en 2013 concluait à une sensibilité de $77 \%$ [IC 95\% (72-81\% )] et une spécificité de 78,9 $\%$ [IC 95\% ( 74-84\% ) ] pour confirmer une bactériémie devant un patient en sepsis ${ }^{(7)}$. Dans le cas précis des infections localisées, les études sont très favorables pour son utilisation pour les infections du tractus respiratoire et exacerbations de BPCO, favorables pour le diagnostic 
des infections urinaires bactériennes et pneumopathies acquises sous ventilation mécanique .Elles sont plus réservées pour les diagnostics d'endocardite ou d'arthrite d'origine bactérienne (1).

Une revue de la littérature de 2016 de van der Does et al étudiant l'intérêt d'une antibiothérapie guidée par la PCT pour l'initiation d'une antibiothérapie aux urgences montrait des résultats insatisfaisants, sauf en cas d'infection respiratoire ${ }^{(8)}$.

Devant ces arguments, ces deux marqueurs habituellement utilisés pour différencier une infection bactérienne d'une autre cause d'hyperthermie et/ou de syndrome inflammatoire sont insuffisants.

\section{Le rapport polynucléaires neutrophiles/lymphocytes (RNL)}

Lors de la première phase d'une infection par un agent bactérien, une des premières réactions immunitaires cellulaires face à l'infection consiste en la mobilisation des polynucléaires neutrophiles de la moelle osseuse au site de l'infection sous l'effet de cytokines proinflammatoires TNF $\alpha$ et IL1, sécrétées par les macrophages situés dans les tissus infectés. Ceci est permis par l'augmentation de perméabilité de l'endothélium vasculaire et la sécrétion de molécules d'adhésion. Le stress engendré par l'infection augmente par la voie hypophysaire le taux de cortisol qui contribue à la démargination des leucocytes. Ces polynucléaires ainsi recrutés joueront un rôle direct de phagocytose de l'agent infectieux sur le site, et entretiendront le phénomène inflammatoire par la sécrétion de cytokines pro-inflammatoires comme l'IL6. Il résulte de ces phénomènes une augmentation du taux de polynucléaires circulants.

La seconde phase de l'inflammation en réponse au stress lié à un stimulus bactérien est le recrutement cellulaire au niveau du site de l'infection et de son système lymphatique (lymphocytes, monocytes) par augmentation de la diapédèse notamment. Le nombre de lymphocytes circulants est diminué, devant une redistribution et une séquestration de la rate vers ces sites. Un second phénomène expliquant la lymphopénie observée est celui de l'apoptose des lymphocytes sous l'effet des cytokines pro-inflammatoires (TNF) mises en cause dans la réaction immunitaire, ainsi que par l'agent bactérien lui-même. 
Chez le sujet âgé, les études évaluant la qualité de la réponse immunitaire cellulaire montrent des modifications à partir de 65 ans, conduisant à une susceptibilité accrue aux infections. Concernant les polynucléaires, l'altération de leur réponse à un stimulus bactérien est liée à un déficit de leur fonction plutôt qu'à un déficit quantitatif. Ce déficit prédomine sur les fonctions de phagocytose des polynucléaires, notamment par la perte de récepteurs nécessaires à cette fonction comme le récepteur CD16, ainsi qu'à une diminution de production des anions superoxydes ${ }^{(8)}$. La réponse lymphocytaire, elle, est modifiée, parfois diminuée. Ceci s'explique par l'involution thymique (au stade de reliquat après 50 ans), et par le défaut de stimulation antigénique au cours du vieillissement, à une diminution des capacités prolifératives et à une modification de sécrétion des cytokines lymphocytaires TH1 (IL2, IFN $\gamma$ ) vers une réponse TH2 (IL4, IL5, IL6, IL12). Les modifications de nombre sont ainsi variables, les lymphocytes voyant leur distribution modifiée (augmentation des T mémoire au dépens des CD4 et CD25+, augmentation des lymphocytes B mémoire au dépens des cellules B naïves et pré-B) ${ }^{(9)}$.

Ainsi, il est permis de penser que le rapport du nombre de neutrophiles sur celui des lymphocytes (RNL) peut être utilisé pour aider au diagnostic d'infection bactérienne, avec une réserve concernant cette utilisation chez le sujet plus âgé. Ceci d'autant plus qu'il concerne un test utilisé de façon courante chez les patients hospitalisés et de faible coût.

Partant de ce constat, ce rapport est étudié de façon prospective par Zahorec et al. en 2001 en réponse à un stress infectieux, chirurgical ou une inflammation systémique dans une population oncologique. L'auteur émet le postulat que ce rapport pourrait être utile pour le diagnostic d'infection bactérienne quelle qu'elle soit, mais également à visée pronostique dans les états infectieux et post-chirurgicaux ${ }^{(11)}$.

De Jager et al. en 2010 étudient la valeur de ce RNL pour le diagnostic de bactériémie à l'admission aux urgences en comparant les NFS des patients aux hémocultures positives à ceux ayant des hémocultures négatives. Ces études montrent des valeurs diagnostiques supérieures à celles des marqueurs usuels que sont la CRP et le nombre de globules blancs (sensibilité de $77,2 \%$, spécificité de $63 \%$ pour un seuil à 10) ${ }^{(12)}$.

Cette hypothèse est émise également par Loonen et al. en 2014. Celui-ci identifie les patients 
bactériémiques parmi les patients admis en état de Syndrome de Réponse Inflammatoire Systémique (SRIS) en réalisant des hémocultures et la recherche de bactéries par PCR. Parmi les patients infectés, le RNL est étudié, et comparé à la CRP, la PCT et un autre marqueur de sepsis, l'urokinase plasminogen activator receptor (suPAR). Les meilleures performances diagnostiques du RNL étaient obtenues pour un seuil à 10 (sensibilité à $85 \%$, spécificité à 51 $\%$ ). Ces valeurs étaient supérieures à la CRP, mais comparables à la PCT et au suPAR, moins utilisés et plus coûteux en pratique ${ }^{(13)}$. L'équipe de Lowsby en 2015 retrouve des valeurs similaires avec une sensibilité à $70 \%$ et une spécificité à $57 \%$, concluant à une possible utilisation de ce ratio en pratique, quand il était associé à des critères cliniques solides pour le diagnostic de bactériémie ${ }^{(14)}$.

Le cut-off proposé était supérieur à 5 pour tout diagnostic d'infection bactérienne et supérieur à 10 pour celui de bactériémie par Gürol et al. en 2014, avec de meilleures performances que la CRP et le nombre de globules blancs. La PCT était utilisée comme gold standard dans cette étude pour affirmer le diagnostic d'infection bactérienne ${ }^{(15)}$.

Laukemann et al confirment récemment ces seuils pour affirmer le diagnostic de bactériémie, avec des résultats similaires, à associer au score clinique proposé par Shapiro ${ }^{(16,17)}$.

Dans le cas des infections localisées, seulement quelques analyses ont été conduites pour confirmer l'origine bactérienne d'une atteinte d'organe. Ainsi, une étude récente de Mentis et al. a calculé les performances du RNL dans le sang et dans le LCR pour le diagnostic de méningite bactérienne. On retrouvait pour le taux sérique une sensibilité de 46,4\% et une spécificité de $83 \%{ }^{(18)}$. Tanriverdi a étudié en 2015 la valeur des marqueurs inflammatoires classiques (CRP, PCT et RNL) pour le diagnostic d'exacerbation de BPCO d'origine bactérienne avec des scores diagnostiques de $61 \%$ pour la sensibilité du RNL, identique à la PCT et $58 \%$ pour la spécificité (vs $67 \%$ pour la PCT) ${ }^{(19)}$. En pédiatrie, Bekdas utilise ce rapport pour différencier une pneumopathie bactérienne d'une pneumopathie virale ${ }^{(20)}$.

Une autre utilisation du RNL, pronostique cette fois, est envisagée pour les infections bactériennes. Ainsi, Turak en 2013 et Bozkay en 2014 trouvaient qu'une valeur élevée de RNL à l'admission était un facteur indépendant de morbi-mortalité intra hospitalière après une endocardite, le premier se concentrant sur les événements neurologiques ${ }^{(21,22)}$. De Jagër montrait ainsi qu'une élévation de ce rapport était associée à une sévérité plus importante des 
pneumopathies communautaires ${ }^{(23)}$. Une étude de 2015 se concentrant sur le pronostic des patients en état de sepsis révélait qu'une valeur basse du RNL à l'admission était corrélée à une survie médiocre à court terme, tandis que son inversion de $\mathrm{J} 1$ à $\mathrm{J} 5$ était prédictive d'une moins bonne survie à long terme ${ }^{(24)}$.

Enfin, les études se multiplient récemment pour démontrer l'utilité de ce rapport pour le pronostic de nombreux cancers (œsophage, pancréas, gastrique), d'atteintes cardiovasculaires et de pathologies inflammatoires comme la spondylarthrite ankylosante et la sarcoïdose. Il est utilisé de façon courante en chirurgie pour le suivi post-opératoire de fractures du col du fémur, appendicectomie,...

Actuellement, nous ne trouvons aucune étude s'intéressant à la valeur diagnostique que pouvait avoir ce rapport devant une fièvre ou une hyperthermie pour le diagnostic d'infection bactérienne, qu'elle soit associée à une bactériémie ou pas à l'admission dans un service de médecine interne. Les études récentes concernaient des populations spécifiques (patients hospitalisés dans un service d'urgences, de soins intensifs ou présentant des pathologies d'organe). Notre étude concerne un service hétérogène particulier, regroupant une grande diversité de patients et de pathologies.

L'objectif de notre étude est de comparer les performances diagnostiques du RNL par rapport aux marqueurs usuels que sont le nombre de globules blancs, de polynucléaires neutrophiles, la CRP et la PCT pour différencier une cause bactérienne d'une autre cause d'hyperthermie et/ou de syndrome inflammatoire chez les patients hospitalisés pour ce motif dans le service de médecine interne.

Le RNL sera également étudié chez les sujets âgés de plus de 75 ans, les phénomènes d'immuno-sénescence pouvant modifier ce rapport.

La mortalité intra-hospitalière et les critères de gravité à l'admission seront recueillis afin d'évaluer la valeur pronostique de ces différents marqueurs. 


\section{MATERIEL ET METHODES}

\section{Patients :}

Les patients ont été inclus de façon rétrospective. Les données ont été recueillies à partir du dossier hospitalier informatisé du patient. Une première analyse rapide des données était effectuée pour chaque patient hospitalisé dans le service de médecine interne du CHU d'Amiens du 01/01/2014 au 31/12/2014 afin de sélectionner les patients susceptibles de rentrer dans l'étude.

Tous les patients présentant à l'admission dans le service une hyperthermie définie par une température corporelle $>38^{\circ}$ et/ou un syndrome inflammatoire défini par un dosage de CRP supérieur ou égal à $20 \mathrm{mg} / \mathrm{L}$ étaient sélectionnés pour l'étude.

Les critères cliniques d'exclusion de l'étude étaient les facteurs susceptibles de modifier l'analyse de la numération formule sanguine et des marqueurs inflammatoires : une néoplasie connue active et/ou en cours de traitement, une pathologie hématologique connue, la grossesse, la séropositivité pour le VIH. Les traitements pouvant modifier les critères de l'analyse comme une chimiothérapie en cours, un traitement immunosuppresseur, une corticothérapie (modification de la numération formule sanguine), ou une antibiothérapie dans les 48 heures précédant l'admission dans le service de médecine interne entrainaient également l'exclusion de l'étude (en raison du risque de négativation des prélèvements).

Parmi les patients inclus dans l'analyse, les données cliniques suivantes étaient recueillies : la date d'admission et durée d'hospitalisation, l'âge, le sexe, le poids et la taille avec calcul du BMI, le statut tabagique (tabagisme actif ou sevrage inférieur à 5 ans).

\section{Diagnostic}

L'analyse des données à partir de l'observation clinique, du courrier de sortie, des courriers de consultation de suivi et des analyses biologiques, microbiologiques et para-cliniques (imagerie, 
anatomopathologie...) par l'investigateur de l'étude définissait le diagnostic final du patient.

Ce ou ces diagnostics étaient classés en différentes catégories suivant leur caractère infectieux ou non. En cas d'infection, les causes bactériennes étaient différenciées des causes virales, fongiques ou parasitaires. En cas de diagnostic non infectieux, on différenciait les pathologies néoplasiques, inflammatoires ou thrombotiques. Les pathologies non identifiées ou les diagnostics incertains étaient classés comme « indéfinis ».

Pour chaque patient inclus, la présence ou l'absence d'hyperthermie était notée à l'admission. Les premières données biologiques à l'arrivée du patient étaient extraites : NFS (taux d'hémoglobine en grammes par dL, Volume Globulaire Moyen en $\mu^{3}$, nombre de plaquettes par $\mathrm{mm}^{3}$, nombre absolu de globules blancs par $\mathrm{mm}^{3}$ dont le nombre de polynucléaires neutrophiles, basophiles ou éosinophiles, le nombre de lymphocytes par $\mathrm{mm}^{3}$, et de monocytes par $\mathrm{mm}^{3}$ ) et les marqueurs inflammatoires usuels la CRP en $\mathrm{mg} / \mathrm{L}$ et la PCT en ng/L quand elle était dosée. Le RNL était calculé à partir de ces données.

L'identification des infections bactériennes était réalisée si possible à partir de critères cliniques et paracliniques précis et validés, en s'appuyant notamment sur les critères de la Société de Pathologies Infectieuses de Langue Française (SPILF) et recommandations internationales.

Ainsi, une infection neuro-méningée était définie par l'association de symptômes neurologiques (céphalées, confusion, signes de localisation à l'examen clinique) et de critères microbiologiques (plus de 10 éléments $/ \mathrm{mm}^{3}$ au niveau du LCR, mise en évidence d'un germe à la culture) ${ }^{(25)}$. Une infection digestive associait des diarrhées, vomissements, douleurs abdominales à une coproculture et/ou des biopsies digestives positives (présence de polynucléaires avec éventuelle mise en évidence de l'agent pathogène) et/ou des signes radiologiques (signes inflammatoires coliques ou intestinaux d'allure infectieuse). Une infection pulmonaire pouvait associer une toux, expectorations purulentes, dyspnée à des signes de pneumopathie alvéolaire ou interstitielle sur la radiographie de thorax ou le TDM thoracique, à des critères biologiques (Examen cytobactériologique des crachats (ECBC), liquide broncho alvéolaire (LBA), prélèvement trachéal protégé (PTP) de bonne qualité présentant plus de 10 polynucléaires neutrophiles $/ \mathrm{mm}^{3}$ et moins de 10 cellules pharyngées $/ \mathrm{mm}^{3}$, avec mise en évidence d'un germe à l'examen direct et/ou à la culture) ${ }^{(26)}$. L'endocardite était définie selon les critères de Duke modifiés ${ }^{(27)}$. Une infection urinaire devait associer des signes fonctionnels 
urinaires (brûlures mictionnelles, pollakiurie, dysurie, douleur au toucher rectal ou l'ébranlement des fosses lombaires) à des critères biologiques (Examen cyto-bactériologique des urines (ECBU) : leucocyturie supérieure à $10 / \mathrm{mm}^{3}$, présence d'un germe en quantité significative (supérieur ou égal à $10^{3} / \mathrm{mm}^{3}$ pour l'Escherichia coli et le Staphylococcus saprophyticus; supérieur ou égal à $10^{3} / \mathrm{mm}^{3}$ chez l'homme pour les autres bactéries ; supérieur ou égal à $10^{4} / \mathrm{mm}^{3}$ chez la femme pour les autres bactéries) ${ }^{(28)}$. Une infection ostéo-articulaire associait des douleurs ou des signes locaux (écoulement, signes inflammatoires) à des critères radiologiques (signes d'ostéite, spondylodiscite...) et microbiologiques sur liquide articulaire ou biopsie osseuse ou disco-vertébrale (présence de polynucléaires, mise en évidence d'un germe). Une infection cutanée était définie par la présence de signes inflammatoires locaux, pouvant être associés à la mise en évidence de bactéries sur les prélèvements locaux, si les conditions de prélèvements étaient favorables (réalisation après lavage au sérum physiologique, prélèvement profond). Le terme d'infection de matériel comprenait des signes locaux en regard du matériel infecté (douleurs, écoulement, désunion de cicatrice) à la positivité de culture du pus prélevé à ce niveau ou du matériel lui-même. La définition d'une infection bactérienne dite « autre » ne correspondant pas à ces critères était laissée à l'appréciation de l'investigateur de l'étude.

En cas d'infection bactérienne avérée, différents éléments étaient recueillis : la réalisation ou non de prélèvement à visée microbiologique, l'identification du germe en cas de positivité, l'existence d'une bactériémie par la positivité des hémocultures. Il était noté la notion de mise sous antibiothérapie avec description de l'antibiothérapie le cas échéant.

\section{Pronostic}

La durée d'hospitalisation était calculée à partir de la date d'admission et de la date de sortie du patient.

Les critères de gravité à l'arrivée dans le service étaient calculés et signalés grâce à l'Indice de Gravité Simplifié Ambulatoire (IGSA) ${ }^{(29)}$ en fonction de l'âge et selon les critères suivants : Pression artérielle systolique, fréquence cardiaque, score de Glasgow, fréquence respiratoire, température. Les patients graves étaient définis par un score IGSA > 4. 
Enfin, le transfert en réanimation pendant l'hospitalisation ou le décès intra-hospitalier était analysé.

\section{Analyse statistique :}

Les données cliniques, biologiques et para-cliniques étaient colligées à partir du dossier informatisé des patients du CHU d'AMIENS (logiciel Dx Care ${ }^{\circledR}$ ) sur le logiciel de base de données EpiDATA ${ }^{\circledR}$.

L'analyse statistique était réalisée à partir de l'extraction des données sur le logiciel SAS $9.2^{\circledR}$.

Les analyses descriptives des populations ont été réalisées. Les variables catégorielles sont exprimées en valeur absolue et pourcentage. Les variables quantitatives normales sont décrites par leur moyenne et écart type, tandis que les variables de répartition non gaussienne le sont par la médiane et intervalle interquartile.

Un calcul des sensibilité, spécificité, valeur prédictive positive et valeur prédictive négative de chaque marqueur a été réalisé, avec détermination de l'intervalle de confiance à $95 \%$. Les valeurs de sensibilité et spécificité ont été exprimées en pourcentage. Les performances opératoires globales des marqueurs ont été comparées au moyen de courbes ROC. Les aires sous la courbe de chaque marqueur ont pu être calculées et comparées, avec un seuil de significativité $p<0.005$. Le seuil de RNL permettant d'obtenir les meilleures sensibilités et spécificité a été recherché au moyen du calcul de l'indice $\mathrm{J}$ de Youden $(\mathrm{J}=\mathrm{Se}+\mathrm{Sp}-1)$.

D’après les études précédentes ${ }^{(12)}$, nous avons pu anticiper une sensibilité du test diagnostique dans ces indications d'environ $70 \%$ (la spécificité étant dans ces études constamment supérieure à la sensibilité). En partant de cette hypothèse, avec une borne inférieure de l'intervalle de confiance de la sensibilité acceptée à $65 \%$, une puissance de $90 \%$, et une erreur alpha de $5 \%$, le nombre de patient à inclure était de 342 .

Le protocole de cette étude a reçu l'approbation de la sous-commission d'évaluation des recherches non-interventionnelles du Comité de Protection des Personnes Nord-Ouest II en date du 11/02/2016. Une lettre d'information a été rédigée pour informer les patients inclus de 
l'analyse de leurs données et du caractère non interventionnel de l'étude. 


\section{RESULTATS :}

\section{Description de la population :}

\section{Critères d'inclusion et d'exclusion :}

Mille quatre cent quatre vingt dix patients ont été hospitalisés dans le service de médecine interne du CHU d'Amiens en 2014. Le critère « hyperthermie et/ou syndrome inflammatoire » était présent à leur arrivée dans le service chez 510 patients, sélectionnés pour l'étude. Un ou plusieurs critères d'exclusion étaient présents chez 171 patients : « néoplasie » $(\mathrm{n}=51)$, «pathologie hématologique » $(\mathrm{n}=22)$, « séropositivité VIH » $(\mathrm{n}=6)$, « grossesse » $(\mathrm{n}=1)$, « traitement immunosuppresseur » $(\mathrm{n}=50)$, « Corticothérapie » $(\mathrm{n}=54)$ ou « antibiothérapie dans les $48 \mathrm{~h}$ précédant l'admission $»(\mathrm{n}=70)$. (Figure 1)

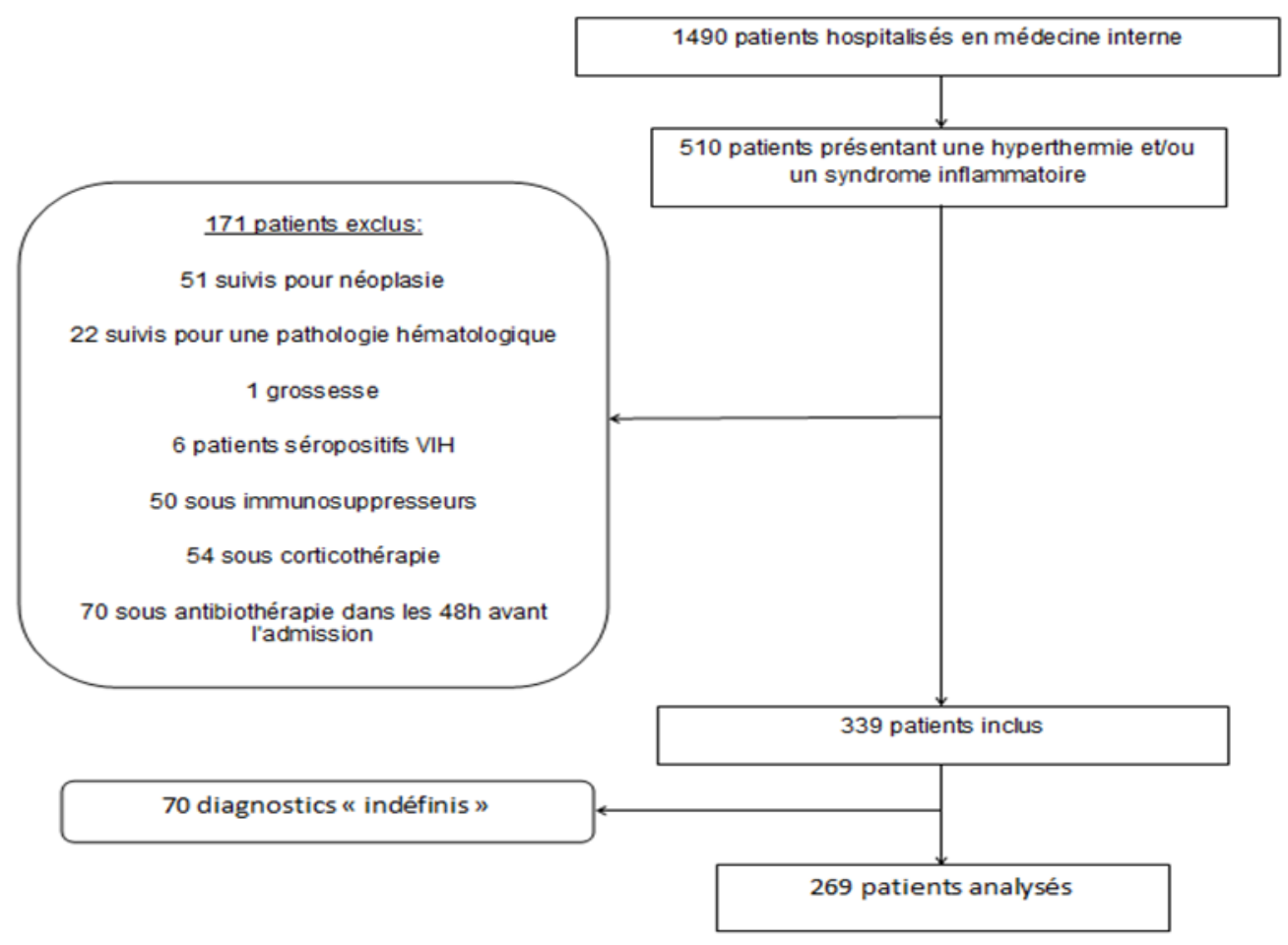

Figure 1 : Description des patients analysés et inclus après analyse des critères d'exclusion. 


\section{Diagnostics de sortie :}

Parmi les diagnostics de sortie expliquant la fièvre et /ou le syndrome inflammatoire, 129 cas étaient en rapport avec une infection bactérienne (38\%), 17 avec une infection virale (5\%), 5 une infection parasitaire $(1,47 \%), 1$ une infection fongique $(0,29 \%), 39$ une pathologie néoplasique $(11,5 \%), 15$ une pathologie thrombotique $(4,42 \%), 53$ une pathologie inflammatoire $(15,63 \%), 10$ une cause dite « autre » $(2,95 \%)$ et 70 patients une cause « indéfinie » $(20,65 \%)$. Seuls les patients ayant une étiologie identifiée étaient retenus pour l'analyse principale $(\mathrm{N}=269)$. Les patients au diagnostic « indéfini » étaient utilisés dans une analyse de sensibilité pour tester la robustesse des résultats..

Les caractéristiques de ces patients sont décrites dans le Tableau 1 en fonction de la cause bactérienne ou non de la fièvre et/ou du syndrome inflammatoire.

Il semblait que les hommes souffraient plus d'infection bactérienne, ainsi que les patients les plus âgés (âge médian à 72 ans [58,5- 82,7] contre 66,3 [44,7- 76,4] pour une étiologie non bactérienne) et les patients fumeurs ( $65 \%$ d'infections bactériennes vs autres diagnostics).

Les patients présentant une infection bactérienne avaient significativement moins de fièvre. Sur le plan biologique, ces patients présentaient une CRP et une PCT à l'admission significativement plus élevées, ainsi que le nombre de globules blancs, de polynucléaires neutrophiles, éosinophiles et basophiles, ainsi qu'un taux d'hémoglobine et de plaquettes inférieurs. 


\begin{tabular}{|c|c|c|c|c|}
\hline \multirow[b]{3}{*}{ Nombre de patients } & \multicolumn{2}{|c|}{ DIAGNOSTIC FINAL } & \multirow{3}{*}{$\mathrm{P}$} & \multirow{3}{*}{$\frac{\text { Total }}{269}$} \\
\hline & Infections bactériennes & Autres étiologies & & \\
\hline & $48 \%$ & $52 \%$ & & \\
\hline Sexe ratio $F / H$ & 1,2 & 0,73 & 0,05 & 0,93 \\
\hline \multicolumn{5}{|l|}{ Age (ans) } \\
\hline Médiane [Q25 \% -Q75 \% ] & $72[58,5-82,7]$ & $66,3[44,7-76,4]$ & 0,001 & $69[51,3-79,4]$ \\
\hline Tabagisme actif & $65 \%$ & $35 \%$ & $<0,001$ & 60 \\
\hline \multicolumn{5}{|l|}{$B M I\left(k g / m^{2}\right)$} \\
\hline Médiane [Q25 \% -Q75 \% ] & $24,6[21,8-29]$ & $24,9[21,5-29]$ & 0,89 & $24,76[21,6-29]$ \\
\hline Fièvre & $38 \%$ & $62 \%$ & $<0,001$ & 105 \\
\hline Syndrome inflammatoire & $48 \%$ & $52 \%$ & 0,68 & 261 \\
\hline \multicolumn{5}{|l|}{$P C T(n g / m L)$} \\
\hline Médiane [Q25 \% -Q75 \% ] & $0,6[0,3-2,1]$ & $0,2[0,1-0,55]$ & 0,012 & $0,39[0,2-1,29]$ \\
\hline \multicolumn{5}{|l|}{$C R P(m g / L)$} \\
\hline Médiane [Q25 \% -Q75 \% ] & $114,6[56-180]$ & $65[32,1-113,9]$ & & $88,7[43,5-145,5]$ \\
\hline \multicolumn{5}{|l|}{ Numération Formule Sanguine. } \\
\hline \multicolumn{5}{|l|}{ Médiane [Q25 \% -Q75 \% ] } \\
\hline Globules Blancs $\left(/ \mathrm{mm}^{3}\right)$ & $12400[9500-16500]$ & 9400 [7150-11400] & $<0,001$ & 10839 [8373-13845] \\
\hline Polynucléaires neutrophiles $\left(/ \mathrm{mm}^{3}\right)$ & $10000[7400-14800]$ & $7100[4850-9200]$ & $<0,001$ & 8491 [6073-11889] \\
\hline Polynucléaires éosinophiles $\left(/ \mathrm{mm}^{3}\right)$ & $0[0-100]$ & $100[0-200]$ & 0,012 & $52[0-152]$ \\
\hline Polynucléaires basophiles $\left(/ \mathrm{mm}^{3}\right)$ & $0[0-0]$ & $0[0-0]$ & $<0,001$ & $0[0-0]$ \\
\hline Lymphocytes $\left(/ \mathrm{mm}^{3}\right)$ & $1100[700-1600]$ & $1400[900-1800]$ & 0,025 & 1301 [804-1704] \\
\hline Monocytes $\left(/ \mathrm{mm}^{3}\right)$ & $600[400-800]$ & $600[450-750]$ & 0,54 & $600[426-774]$ \\
\hline Hémoglobine (g/dL) & $12,9[11,5-13,9]$ & $12,6[10,9-14]$ & 0,38 & $12,7[11,2-14]$ \\
\hline Plaquettes $\left(/ \mathrm{mm}^{3}\right)$ & $248000[178000-321000]$ & $282500[203500-366000]$ & 0,007 & 265955[191271-344420] \\
\hline
\end{tabular}




\section{Description des étiologies non bactériennes :}

Les étiologies non bactériennes étaient réparties selon leur cause : virale $(\mathrm{N}=17)$, parasitaire $(\mathrm{N}=5)$, fongique $(\mathrm{N}=1)$, néoplasique $(\mathrm{N}=39)$, thrombotique $(\mathrm{N}=15)$, inflammatoire $(\mathrm{N}=53)$, ou autres $(\mathrm{N}=10)$.

Les étiologies virales documentées étaient une infection à coxsakie virus, 2 infections herpétiques, une encéphalite zostérienne, une hépatite B et deux hépatites E. Les autres infections virales non documentées étaient des méningites virales, bronchites aigües et gastro entérite aigüe.

Les étiologies parasitaires étaient 4 accès palustres à Plasmodium falciparum, et un abcès amibien hépatique.

L'étiologie fongique était une vaginite à Candida.

Les étiologies néoplasiques regroupaient des pathologies hématologiques (2 amyloses AL, 5 lymphomes et une leucémie aigüe myéloïde), et des tumeurs solides ( 3 localisations prostatiques, 5 localisations pulmonaires, 2 localisations ORL, 7 localisations hépatiques, 5 localisations coliques, une localisation gastrique, une localisation cérébrale, une localisation utérine, une localisation ovarienne, une localisation mammaire, et une tumeur pyélique, un mélanome, un liposarcome). Chez un patient, une néoplasie au stade métastatique était découverte sans primitif évident. Cinq de ces affections étaient diagnostiquées à un stade métastatique.

Les étiologies thrombotiques étaient d'origine artérielle (notamment infarctus cérébral et colite ischémique) et veineuse (maladie thrombo-embolique veineuse et thrombose portale principalement).

Les étiologies inflammatoires les plus fréquentes étaient représentées par des pancréatites aigües (10 patients), des pseudo-polyarthrites rhizoméliques isolées (8 patients), des maladies de Horton (7 patients), des arthrites micro-cristallines (5 patients) et des maladies inflammatoires chroniques de l'intestin (5 patients).

Les autres causes étaient principalement des hématomes, une cirrhose et une sténose peptique de l'œsophage.

\section{Infections bactériennes :}

\section{Localisation :}


Parmi les 129 infections bactériennes, 39,4\% étaient de localisation pulmonaire, 23,6\% de localisation urinaire, 14,2\% de localisation digestive, 7,9\% de localisation « autre », 7,1\% de localisation cutanée, 5,5\% de localisation ostéo-articulaire, et $0.8 \%$ de localisation neuro-méningée et sur matériel.

Les localisations « autres » concernaient une infection sur pontage aorto-coronarien, trois infections ORL, une tuberculose ganglionnaire et colique, une endométrite à Haemophilus influenzae, un abcès du psoas à Streptococcus pyogenes, une endocardite aortique à Staphylococcus aureus compliquée de spondylodiscite et une tularémie.

\section{Documentation microbiologique :}

Chez ces 129 patients présentant une infection bactérienne, 96 ont bénéficié d'hémocultures, ce qui a permis de mettre en évidence 23 bactériémies.

Cent soixante-quinze prélèvements à visée microbiologique ont été réalisés. Un ou plusieurs agents microbiens ont été mis en évidence sur 71 de ces prélèvements. La répartition de ces examens et leur positivité est décrite dans la figure 2. 


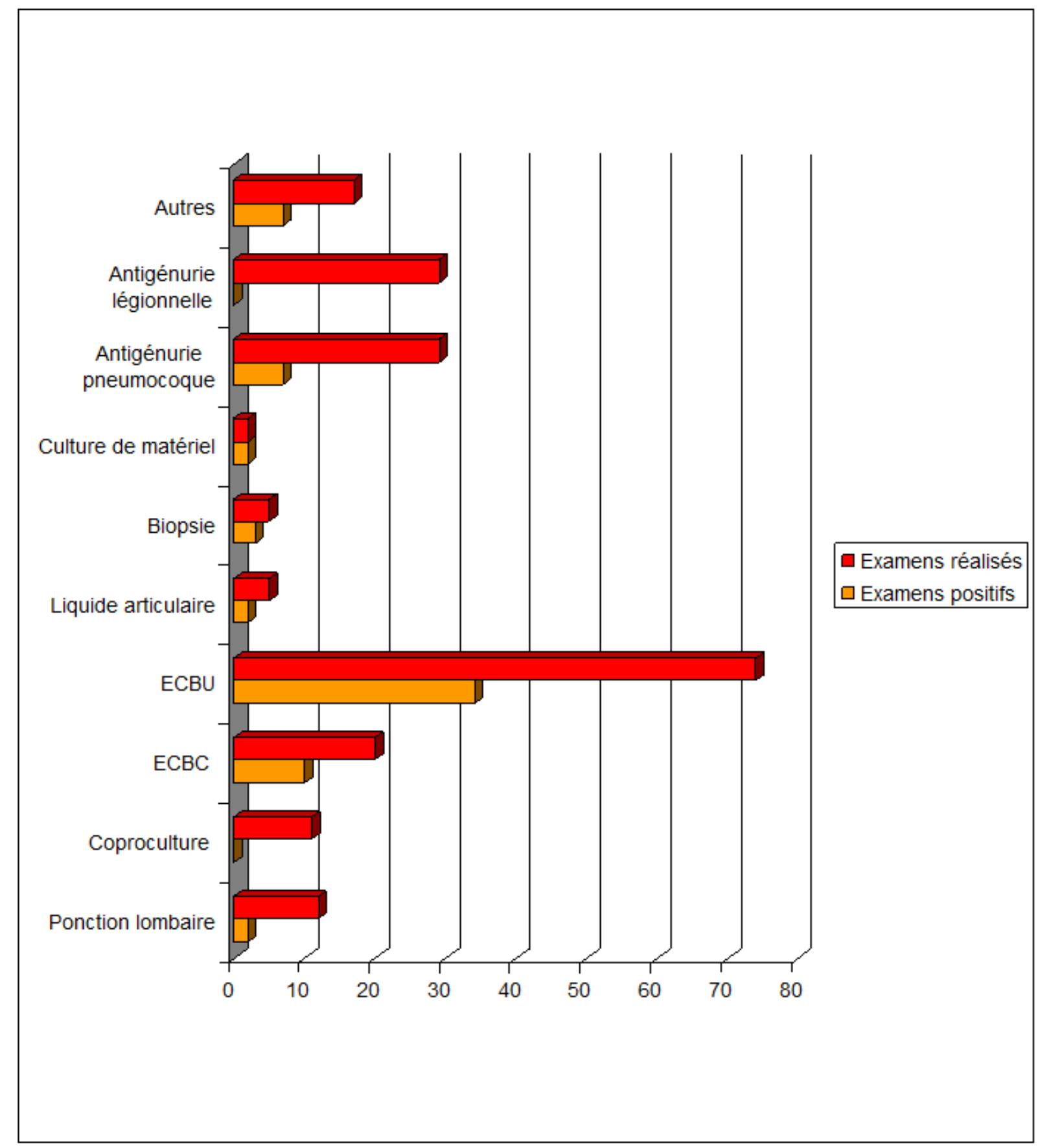

Figure 2 : Description des examens microbiologiques réalisés et leur positivité. 
Les germes les plus souvent retrouvés sont décrits dans le tableau 2. Les bacilles Gram négatifs étaient les germes les plus fréquents (64,8 \%), avec 56,3\% d'Escherichia coli (soit 36,5\% sur tous les germes identifiés). Les cocci Gram positifs constituaient $28,4 \%$ de tous les prélèvements positifs, les cocci Gram négatifs 2,7 \% et les bacilles Gram positif 2,7 \% .

\begin{tabular}{|c|c|}
\hline Micro organismes : & Nombre de patients : \\
\hline COCCI GRAM + & 21 \\
\hline \multicolumn{2}{|l|}{ Streptococcus } \\
\hline pneumoniae & 5 \\
\hline pyogenes & 3 \\
\hline agalactiae & 1 \\
\hline gordonii & 1 \\
\hline \multicolumn{2}{|l|}{ Staphylococcus } \\
\hline aureus & 6 \\
\hline epidermidis & 2 \\
\hline Enterococcus faecalis & 3 \\
\hline COCCI GRAM - & 2 \\
\hline \multicolumn{2}{|l|}{ Neisseria } \\
\hline gonorrhoeae & 1 \\
\hline meningitidis & 1 \\
\hline BACILLE GRAM + & 2 \\
\hline Corynebacterium striatum & 1 \\
\hline Clostridium difficile & 1 \\
\hline BACILLE GRAM - & 48 \\
\hline Escherichia coli & 27 \\
\hline \multicolumn{2}{|l|}{ Citrobacter } \\
\hline koseri & 1 \\
\hline freundii & 1 \\
\hline \multicolumn{2}{|l|}{ Klebsiella } \\
\hline pneumoniae & 5 \\
\hline oxytoca & 1 \\
\hline Haemophilus influenzae & 3 \\
\hline Pseudomonas aeruginosa & 4 \\
\hline Bacteroides fragilis & 1 \\
\hline Morganella morgani & 1 \\
\hline Francisella tularensis & 1 \\
\hline Enterobacter cloacae & 1 \\
\hline \multicolumn{2}{|l|}{ Salmonella } \\
\hline bovis & 1 \\
\hline paratyphii & 1 \\
\hline Chlamydia trachomatis & 1 \\
\hline
\end{tabular}




\section{Antibiothérapie :}

Une antibiothérapie était prescrite en mono ou pluri-thérapie chez 123 patients. Les pathologies non traitées étaient une iléite d'allure infectieuse non documentée et d'évolution spontanément favorable, une infection urinaire sur globe vésical d'évolution favorable après sondage vésical chez une patiente de 77 ans, une diverticulite sigmoïdienne en cours de cicatrisation et d'évolution spontanément favorable, un abcès du psoas traité après transfert en chirurgie pour drainage, un abcès dentaire traité par voie locale uniquement et une pneumopathie du lobe supérieur droit qui sera traitée après la sortie du patient.

Les antibiotiques les plus souvent prescrits étaient les $\beta$ lactamines $(\mathrm{N}=114)$, dont les céphalosporines de $3^{\text {e }}$ génération $(\mathrm{N}=51)$, l'association amoxicilline/acide clavulanique $(\mathrm{N}=40)$, l'amoxicilline seule $(\mathrm{N}=12)$, l'association piperacilline/tazobactam $(\mathrm{N}=7)$, l'oxacilline $(\mathrm{N}=2)$, l'aztreonam $(\mathrm{N}=1)$ et la ceftazidime $(\mathrm{N}=1)$. Les autres antibiotiques prescrits étaient les fluoroquinolones $(\mathrm{N}=28)$, le métronidazole $(\mathrm{N}=16)$ et les aminosides $(\mathrm{N}=13)$, la rifampicine $(\mathrm{N}=6)$, les macrolides $(\mathrm{N}=3)$, le linezolide $(\mathrm{N}=3)$, glycopeptides $(\mathrm{N}=3)$, la daptomycine $(\mathrm{N}=2)$. Une seule prescription était relevée pour les carbapénèmes, la pristinamycine, le trimethoprime-sulfamethoxazole, la clindamycine, l'ethambutol, la pyrazinamide et l'isoniazide.

\section{II. $\underline{\underline{\text { RNL : }}}$}

\section{Calcul du RNL selon les étiologies :}

La médiane et l'intervalle interquartile du RNL à la numération formule sanguine de l'admission étaient calculés et classés en fonction des étiologies. (Tableau 3 et Figure 3). Le RNL était statistiquement différent dans les différentes étiologies ( $\mathrm{p}<0,0001$ Test de Kruskall-Wallis). 


\begin{tabular}{lcc}
\hline \multicolumn{1}{c}{ Etiologie } & Nombre de patients & RNL médian $(\mathrm{Q} 25 \%-\mathrm{Q} 75 \%)$ \\
\hline Bactérienne & 129 & $9,59(5,47-16,7)$ \\
Virale & 17 & $4,1(3,54-7,57)$ \\
Parasitaire & 5 & $7,5(5,38-11,33)$ \\
Fongique & 1 & $2(2-2)$ \\
Néoplasique & 39 & $6,5(4,07-10)$ \\
Thrombotique & 15 & $5,57(3,73-10,57)$ \\
Inflammatoire & 53 & $5,06(3,05-7,83)$ \\
Autres & 10 & $4,71(2,64-583)$ \\
\hline
\end{tabular}

Tableau 3 : RNL selon l'étiologie de l'hyperthermie et/ou syndrome inflammatoire.

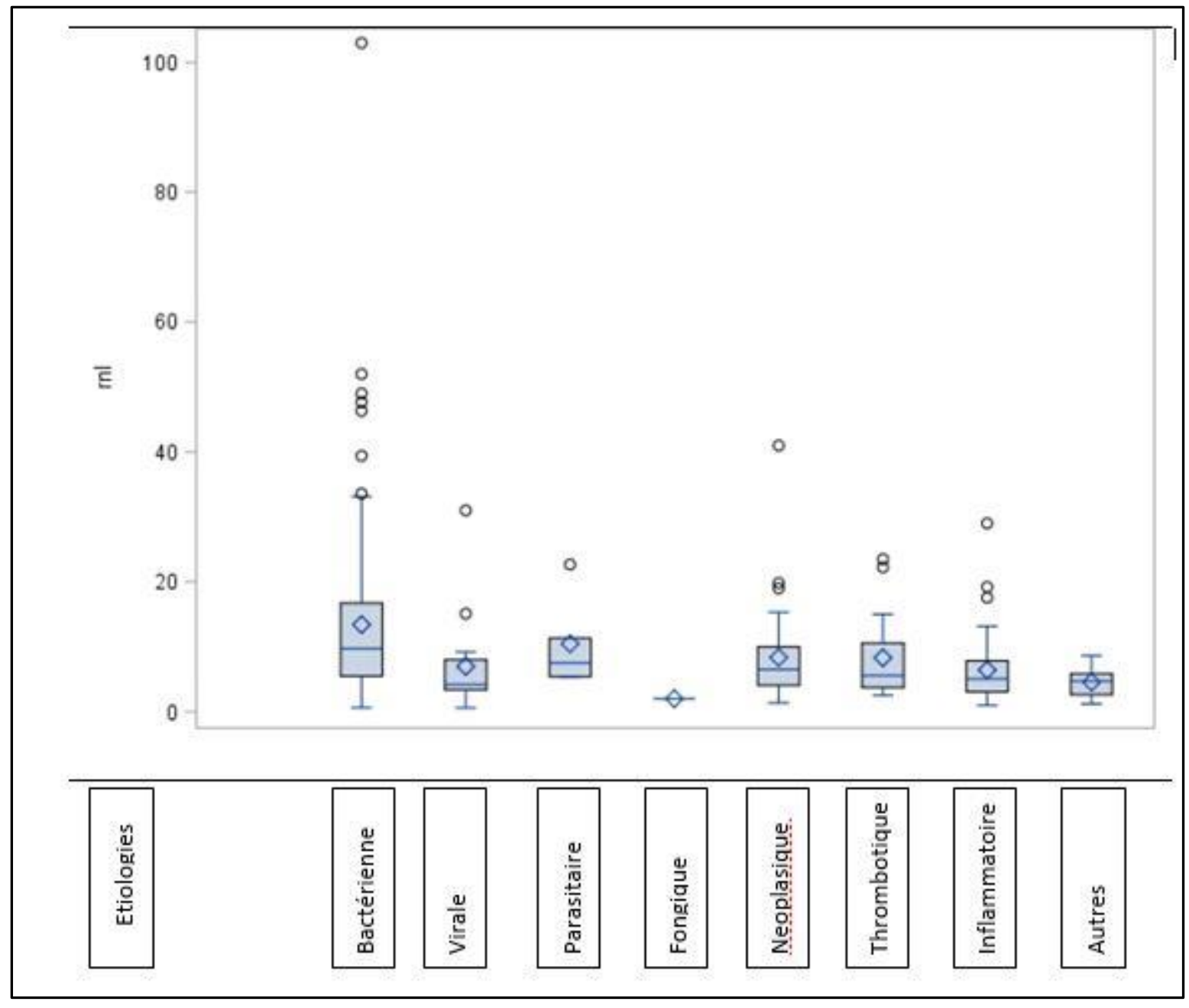

Figure 3 : RNL en fonction de l'étiologie.

Les patients présentant une infection bactérienne avaient un RNL significativement plus élevé que les autres : 9,58 [5,47-16,70] versus 5,38 [3,40-8,70], (p<0,0001, test de Wilcoxon). (Figure 4) 


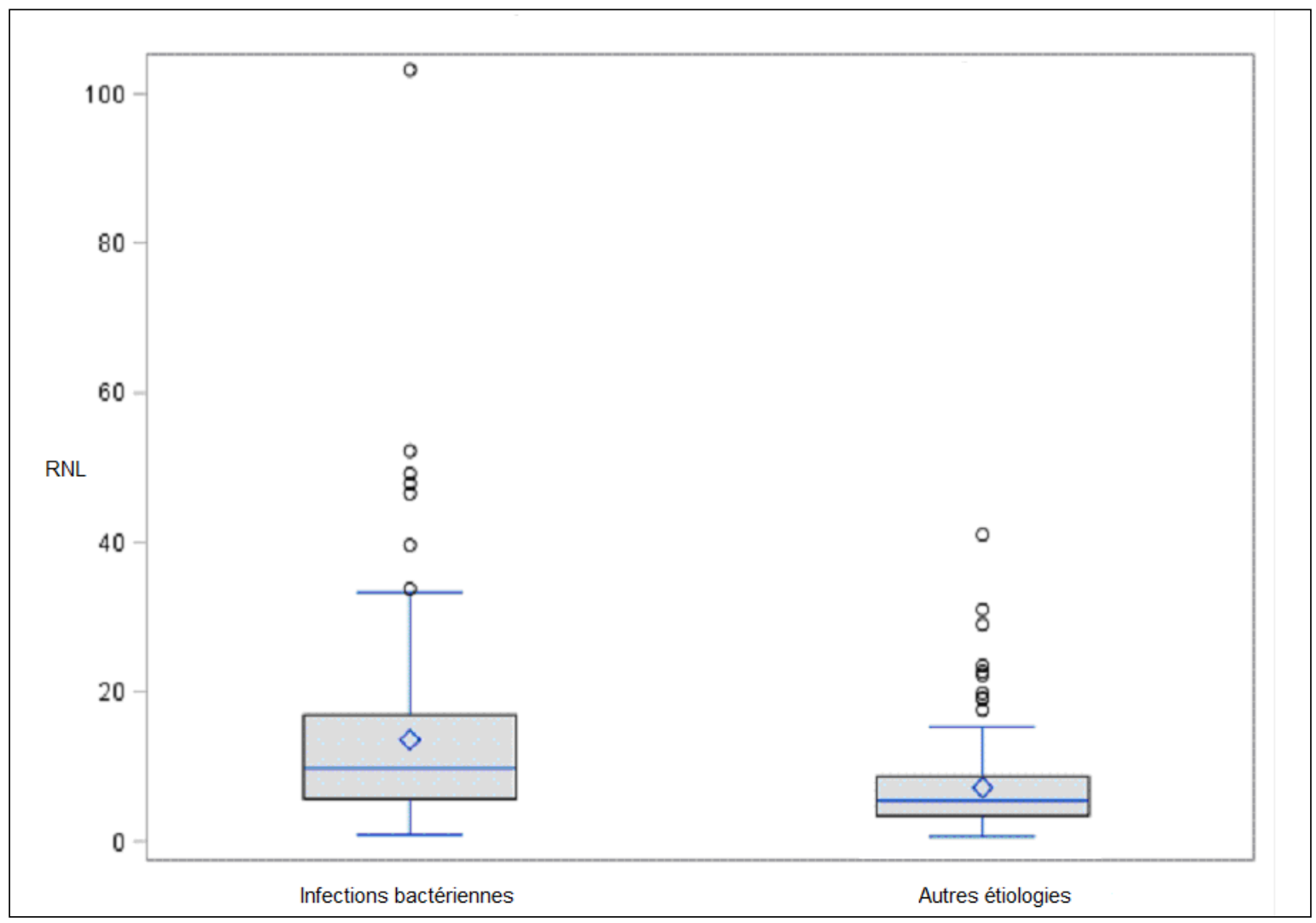

Figure 4 : Comparaison du RNL en cas d'infection bactérienne ou non. Test de Wilcoxon, $p<0,0001$

Le calcul de l'indice de Youden appliqué à ces valeurs permettait d'obtenir un seuil optimal du RNL à 8,2 .

A ce seuil, le calcul des performances diagnostiques du RNL pour le diagnostic d'infection bactérienne permettait d'obtenir une sensibilité de $61 \%$ [IC $95 \%$ (52\%-69\%)], une spécificité de $73 \%$ [IC $95 \%(66 \%-81 \%$ )], une valeur prédictive positive de $67 \%$ [IC $95 \%$ (59 \% -76\% )] et une valeur prédictive négative de $67 \%$ [IC $95 \%(60 \%-75 \%)$ ].

La courbe ROC était tracée pour déterminer les performances diagnostiques du test. L'aire sous la courbe était calculée à 0,69 [IC $95 \%(0,63-0,76)$ ] pour le RNL pour le diagnostic d'infection bactérienne versus autres causes de fièvre et/ou syndrome inflammatoire.

\section{Performances diagnostiques des marqueurs usuels :}

Les calculs de sensibilité et spécificité pour le diagnostic d'infection bactérienne de la CRP, de la PCT, du nombre de globules blancs et de polynucléaires neutrophiles étaient réalisés et la courbe ROC tracée pour chaque marqueur. 
Pour la CRP, l'aire sous la courbe (AUC) était calculée à 0,61 [IC $95 \%(0,46-0,77)$; pour la PCT, l'AUC était de 0,70 [IC $95 \%(0,56-0,84)$ ] ; pour le nombre de globules blancs de 0,67 [IC $95 \%$ $(0,52-0,81)]$; pour le nombre de polynucléaires neutrophiles de 0,70 [IC $95 \%(0,55$ - 0,84)] ; pour le nombre de polynucléaires éosinophiles de 0,60 [IC $95 \%$ ( 0,46 - 0,75)]

Aucune différence statistiquement significative n'était observée entre les AUC des courbes ROC des différents marqueurs. (Figure 5)

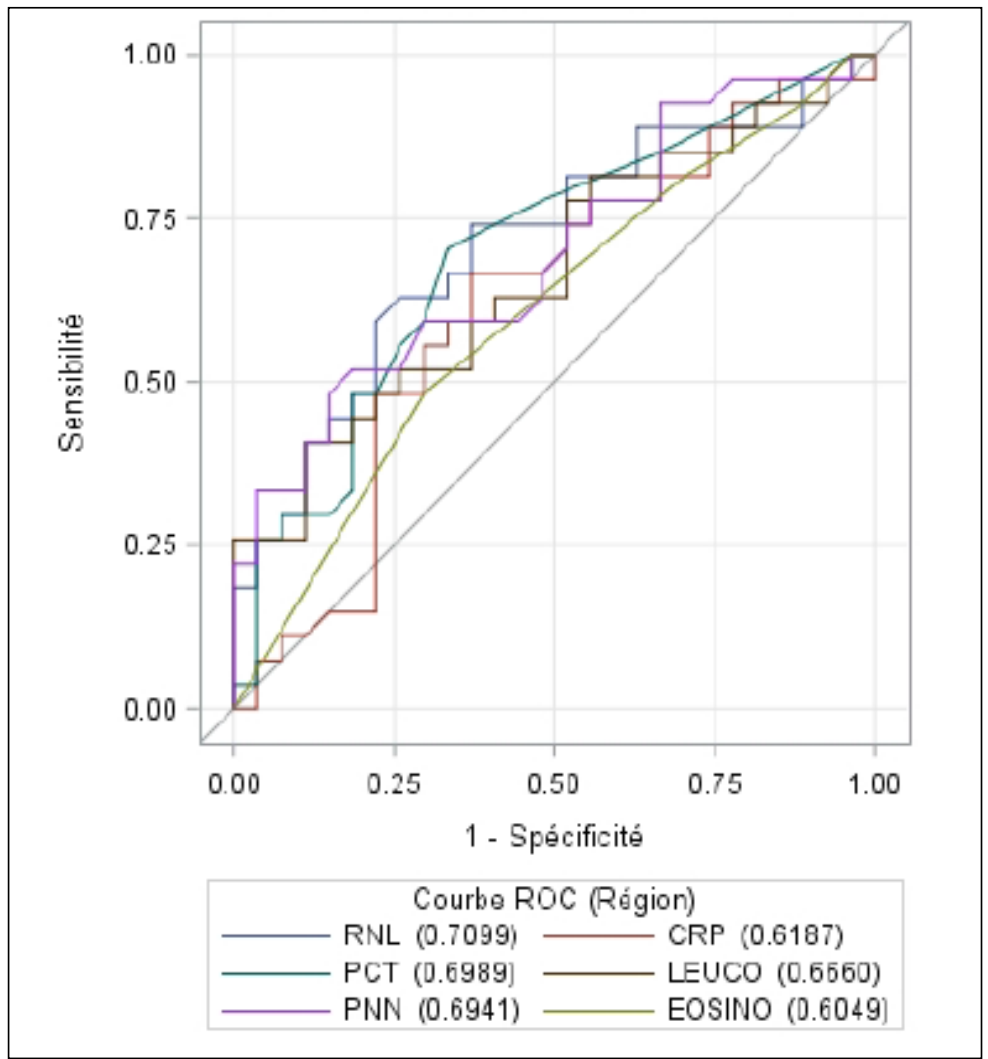

Figure 5 : Comparaison des courbes ROC de chaque marqueur

\section{Analyse de sensibilité :}

Une étude de sensibilité a été réalisée en incluant dans l'analyse les patients au diagnostic final indéterminé. Le RNL médian de ces patients était de 5,13 [3,38-9,43].

Les performances diagnostiques du RNL pour le diagnostic d'infection bactérienne ne s'en trouvaient pas significativement modifiées.

La courbe ROC pour le RNL retrouvait alors une AUC à 0,7 [IC $95 \%(0,64-0,76)]$, sensiblement similaire à celle calculée pour le RNL excluant les patients au diagnostic «indéfini ». $\mathrm{Au} \quad$ seuil de 7,7, les valeurs de sensibilité et spécificité étaient respectivement de $62 \%$ [IC95\% (54-70)] et 
$69 \%$ [IC95 \% (63-76)]. La valeur prédictive positive était de $56 \%$ [IC95 \% (47-64)] et la valeur prédictive négative de $75 \%$ [IC95\% (69-85)].

\section{Performances du RNL pour le diagnostic de bactériémie.}

Les mêmes calculs de sensibilité et spécificité étaient établis pour le diagnostic d'infection bactérienne avec bactériémie chez les 339 patients, tous diagnostics confondus.

Le calcul de l'indice de Youden montrait un seuil optimal du RNL à 10,65. Les valeurs de sensibilité à ce niveau était de $67 \%$ [IC $95 \%$ (48 \% -84 \% )], de spécificité de $74 \%$ [IC $95 \%$ (69\%-79\% )], la valeur prédictive positive de $16 \%$ [IC $95 \%$ (9\%-24\% )] et la valeur prédictive négative de $96 \%$ [IC $95 \%(94 \%-99 \%)$ ].

L'aire sous la courbe ROC tracée à ce seuil était de 0,7 [IC $95 \%(0,58-0,83)]$.

\section{Cas des patients âgés de plus de 75 ans :}

\section{Description de la population :}

Quatre-vingt-quinze patients étaient âgés de plus de 75 ans. On établissait pour 56 de ces patients le diagnostic d'infection bactérienne, pour 12 de pathologie néoplasique, pour 5 de pathologie thrombotique, pour 17 de pathologie inflammatoire, et pour 5 de pathologies « autres ».

\section{Calcul du RNL :}

Le calcul du RNL médian et de l'intervalle interquartile pour ces patients était de 11,69 [7,79-16,68] pour les patients ayant une infection bactérienne, 5,79 [4,64- 9,28] pour les pathologies néoplasiques, 10,57 [9,71-15] pour les pathologies thrombotiques, 6,14 [4,04- 8,06] pour les causes inflammatoires et 5,83 [5,29-7,92] pour les autres causes ( $p<0,0002$, test de Kruskal-Wallis). (Figure 6) 


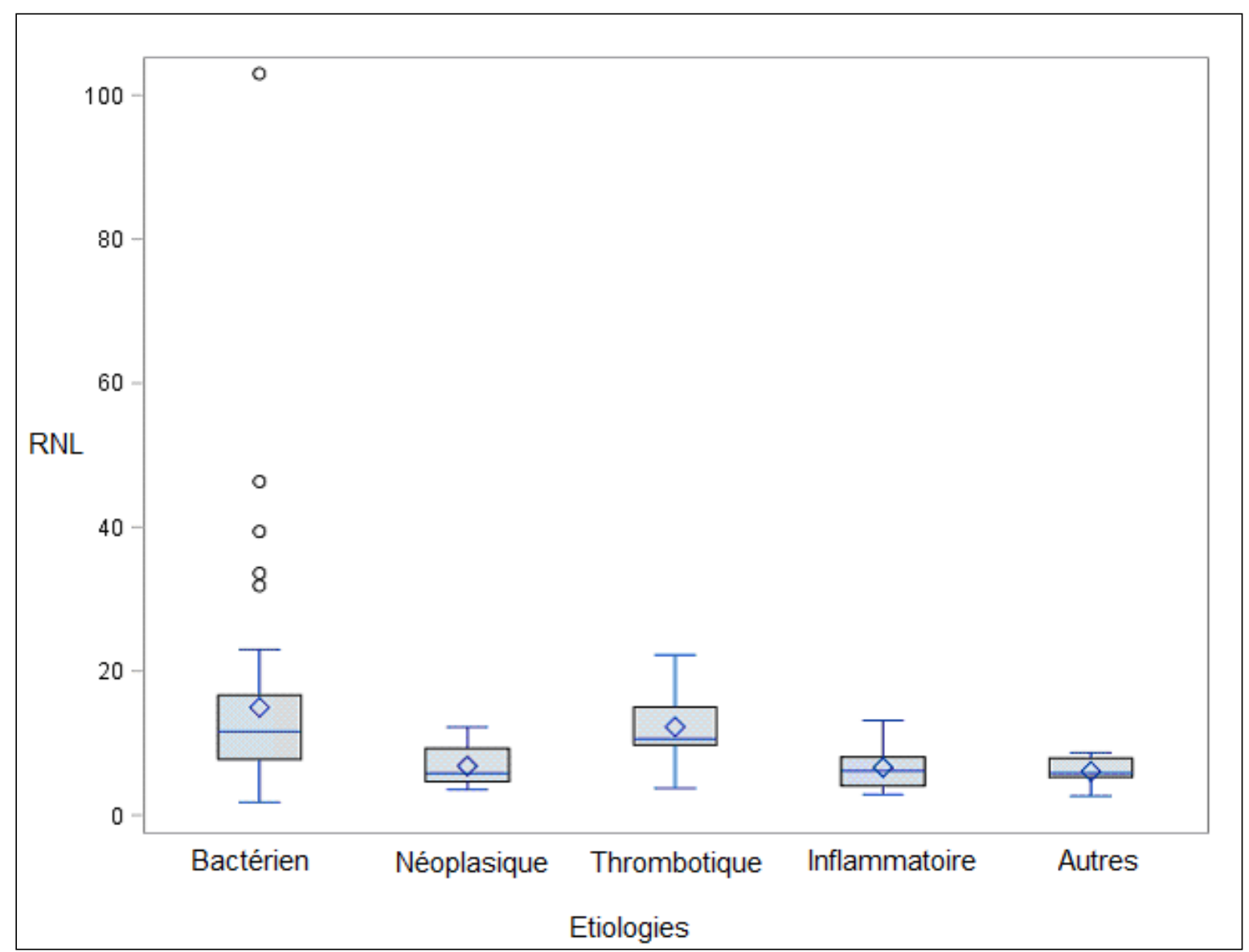

Figure 6 : Comparaison du RNL en fonction des étiologies chez les sujets âgés de plus de 75 ans. Test de Kruskal-Wallis, $p<0,0002$.

Les patients âgés de plus de 75 ans présentant une infection bactérienne avaient un RNL significativement plus élevé : 11,6 [7,75-16.66] versus $6,14[4,46-9,27](\mathrm{p}<0,0001$, test de Wilcoxon). (Figure 7) 


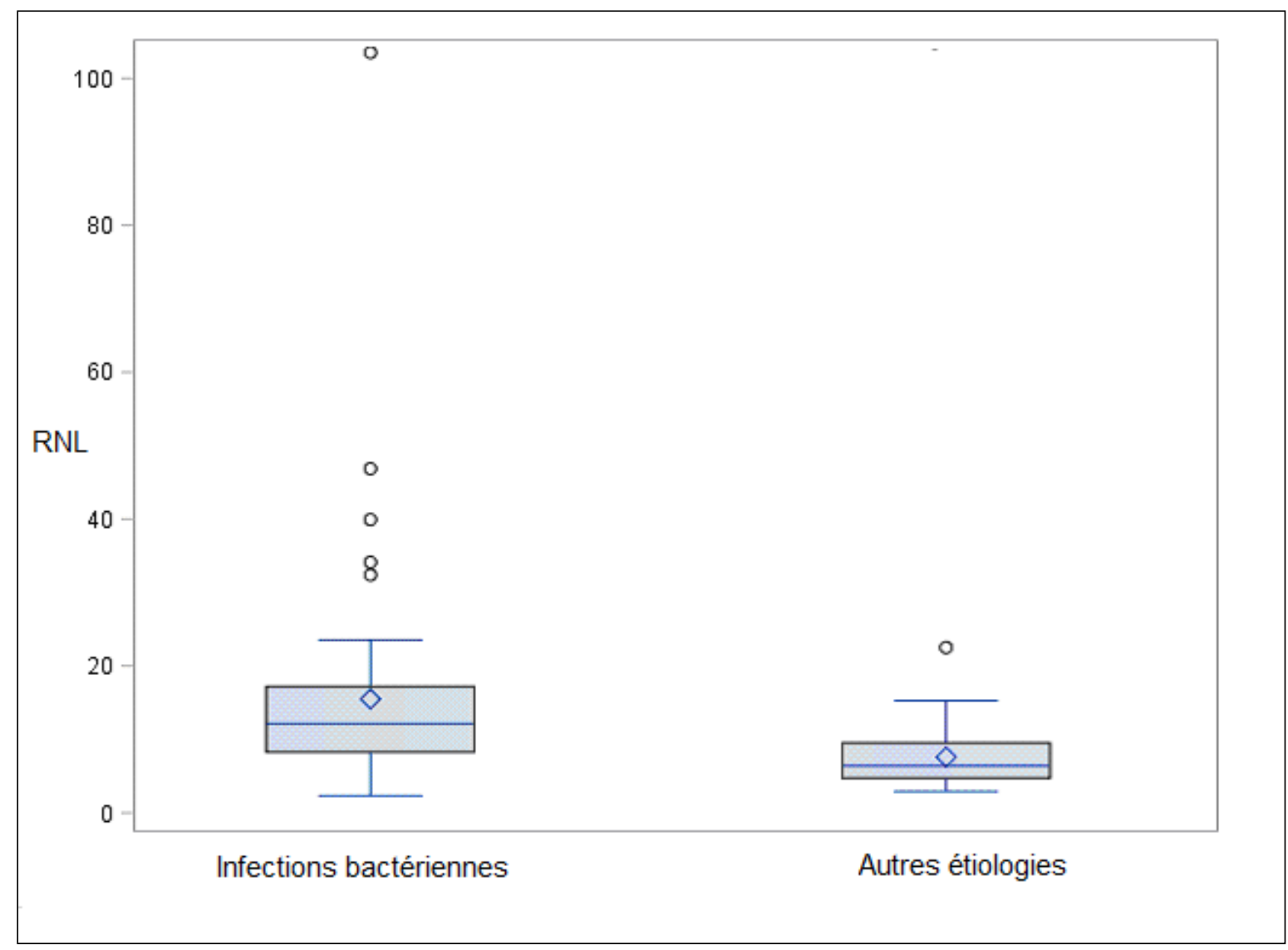

Figure 7 : Comparaison du RNL en cas d'infection bactérienne ou non chez les sujets âgés de plus de 75 ans. Test de Wilcoxon, $p<0,0001$.

Le calcul de l'indice de Youden établissait un seuil optimal à 15,08 pour le diagnostic d'infection bactérienne sans effet seuil très franc. A ce seuil, la sensibilité était de $57 \%$ et la spécificité de $87 \%$.

La courbe ROC tracée pour le RNL chez le sujet de plus de 75 ans met en évidence une AUC de 0,76 [IC $95 \%(0,66-0,86)]$.

\section{Comparaison des performances à celles de la CRP :}

Les mêmes valeurs ont été calculées pour la CRP chez le sujet âgé, retrouvant une AUC de 0,62 [IC $95 \%(0,51-0,74)]$. Les deux courbes ROC (CRP et RNL chez le sujet âgé) ont été comparées. Toutefois, cette différence n'était pas statistiquement significative. (Figure 8) 


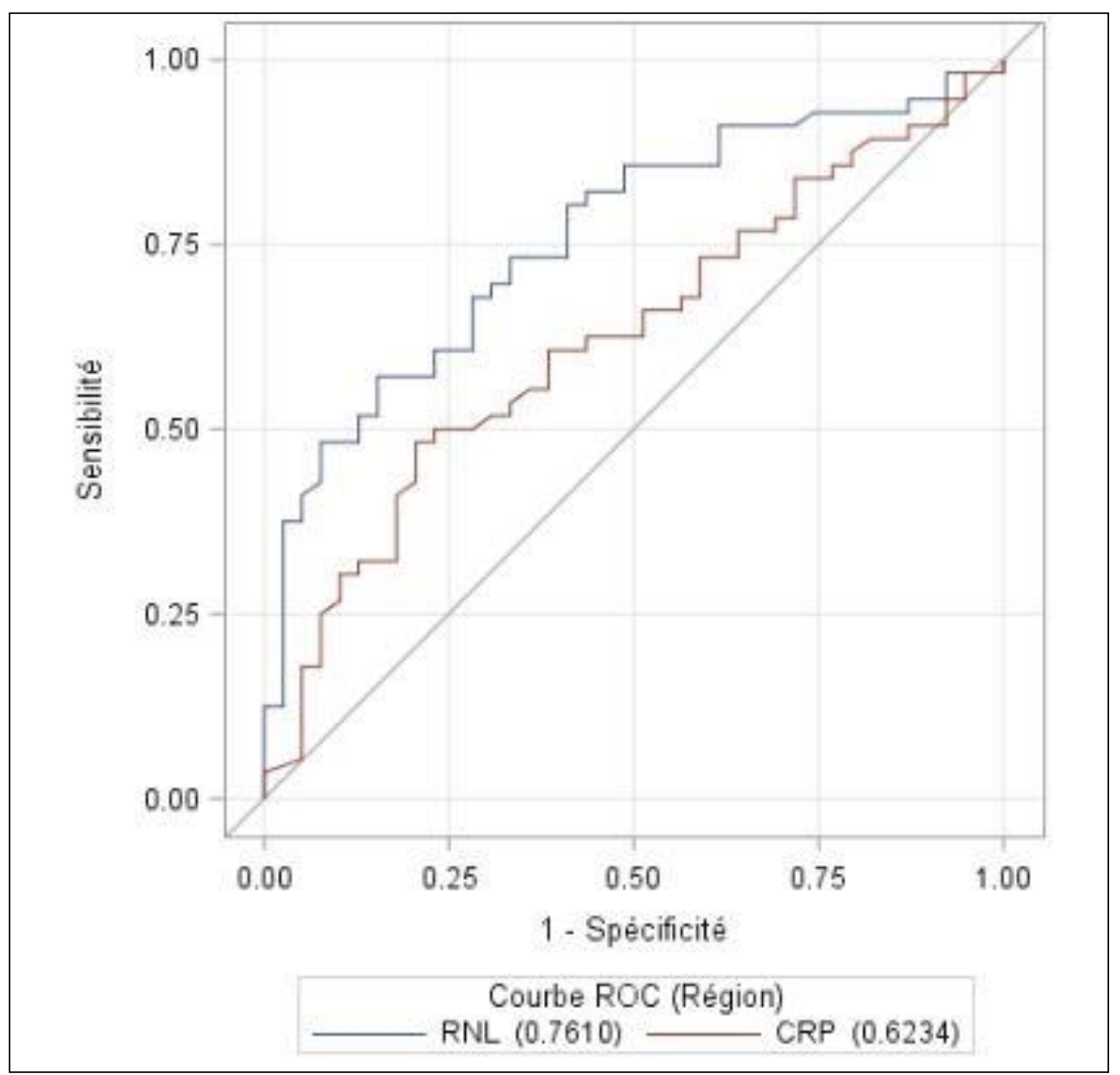

Figure 8 : Comparaison des courbes ROC du RNL et CRP pour les sujets de plus de 75 ans

\section{RNL et pronostic :}

La notion de sévérité était établie chez les patients présentant une infection bactérienne par la présence de critères de gravité à l'admission, ou de décès pendant l'hospitalisation, ou de transfert en réanimation durant le séjour.

Un ou plusieurs critères de gravité étaient notés à l'admission de 17 patients dans le service (13,2\% ), 6 sont décédés pendant l'hospitalisation $(4,7 \%), 5$ ont été transférés en réanimation $(3,8 \%)$.

Le RNL médian de ces patients présentant des critères de gravité à l'admission était de 7,8 [5,4-19] contre 9,2 [5,5-16,6] pour ceux qui n'en présentaient pas. Les patients décédés avaient un RNL médian à 7,3 [6,7-8,6] contre 10 [5,3-17] pour les patients ayant survécu. Les patients ayant été transférés en réanimation avaient un RNL médian à 6,8 [4,8-7,8] contre 9,9 [5,5-16,7] pour ceux n'ayant pas été transférés.

$\mathrm{Au}$ total, les patients « sévères » $(\mathrm{N}=22)$ avaient un $\mathrm{RNL}$ médian calculé à $7,8[5,4-17,4]$ contre 
10 [5,5-16,7] chez les autres ( $\mathrm{p}=0,71$, test de Wilcoxon). Un RNL élevé à l'admission n'apparaissait pas comme un marqueur de sévérité chez les patients présentant une infection bactérienne. 


\section{DISCUSSION :}

\section{Résultats :}

La physiopathologie de l'infection bactérienne suggérait que le rapport neutrophiles sur lymphocytes (RNL) pouvait apporter une aide supplémentaire à l'orientation diagnostique chez un patient hospitalisé pour fièvre et/ou syndrome inflammatoire, la performance des marqueurs habituels (CRP, PCT, nombre de globules blancs et de polynucléaires neutrophiles) étant jugée insuffisante. La mobilisation des polynucléaires neutrophiles sur le site de l'infection, ainsi que la redistribution lymphocytaire et leur apoptose sous l'influence des cytokines pro-inflammatoires, du stress cortisonique et l'agent bactérien lui-même constituent la première ligne de défense immunitaire dans le cadre de ces infections.

Dans notre étude, chez tous les patients hospitalisés en médecine interne pour fièvre et/ou syndrome inflammatoire, le RNL était plus élevé dans les infections bactériennes par rapport aux causes non bactériennes (virales, parasitaires, mycologiques, thrombotiques, néoplasiques ou inflammatoires), et cela de façon statistiquement significative.

Pour un seuil à 8,2, on trouvait une sensibilité à $61 \%$, et une spécificité de $73 \%$. L'aire sous la courbe ROC était de 0,69 [IC $95 \%(0,36-0,76)]$. Cette valeur était supérieure à celle de la CRP, du nombre de globules blancs et de polynucléaires éosinophiles, inférieure à celle de la PCT et du nombre de polynucléaires neutrophiles mais de façon non significative.

Par contre le RNL s'avérait décevant pour identifier les patients sévères, parmi les patients présentant une infection bactérienne. Les mêmes résultats étaient observés pour le diagnostic de bactériémie.

Les phénomènes d'immuno-sénescence (diminution du nombre de lymphocytes, altération de la réponse neutrophilique) laissaient supposer que ce rapport pouvait être modifié chez les sujets âgés. Notre étude montre que le RNL reste significativement plus élevé lors d'une infection bactérienne chez les patients de plus de 75 ans admis pour fièvre et/ou syndrome inflammatoire.

\section{Gold standard :}

Le gold standard de notre étude était la définition d'une infection bactérienne, établie par le praticien 
en charge du patient sur le compte rendu de sortie, à l'issue de l'hospitalisation et à la lumière des différents examens cliniques et para cliniques réalisés. Ce diagnostic de sortie était validé par la relecture du dossier complet par le responsable scientifique, prenant en compte les données ultérieures à la sortie d'hospitalisation (consultations de contrôle, examens biologiques et d'imagerie de contrôle, etc.) Il est donc soumis à un biais de classement, malgré le respect de définitions standardisées et validées. Le praticien en charge du patient, ainsi que le responsable scientifique établissaient en effet leur diagnostic final en raisonnant notamment sur les données biologiques comprenant la NFS à l'admission du patient, et plus précisément le nombre de polynucléaires neutrophiles et de lymphocytes. La définition du gold standard implique donc la prise en compte de critères comprenant les composants du rapport neutrophiles sur lymphocytes et pouvant constituer un biais d'incorporation.

Ces difficultés à identifier formellement les infections bactériennes étaient aussi liées à la population étudiée constituée de patients hospitalisés dans un service de médecine interne, où la réalisation de prélèvements peut être soumise à des contraintes de réalisation (examens invasifs par exemple).

Les études précédentes testant le RNL s'appuyaient d'abord sur des critères microbiologiques pour établir leur gold standard, pouvant exclure les infections bactériennes non documentées, fréquentes dans notre population de patients ${ }^{(12-14)}$. Loonen et de Jager utilisaient les hémocultures pour affirmer le diagnostic de bactériémie, leur étude se concentrant sur les patients suspects de ce diagnostic (présentant des critères de SRIS et ayant bénéficié d'hémocultures à l'admission pour Loonen et « suspects de bactériémie » pour de Jager) à leur admission dans un service d'urgences. Ce recueil était rétrospectif et concernait donc une population de patients ciblée (admise dans un service d'urgences, et suspecte de bactériémie) ${ }^{(13)}$.

Gürol utilisait la PCT comme gold standard pour le diagnostic d'infection bactérienne. Le type d'infection (localisée ou systémique) était défini selon le taux de PCT dosé à l'admission. Ce marqueur étant comparé à celui du RNL, et ses performances limitées, nous n'avons pas retenu ce seul marqueur comme permettant de diagnostiquer les infections bactériennes ${ }^{(15)}$. Dans notre travail, nous démontrons d'ailleurs que les performances diagnostiques de la PCT et du RNL sont similaires pour le diagnostic d'infection bactérienne.

Peu d'études se sont intéressées aux infections bactériennes localisées. La première étude s'interrogeant sur la valeur diagnostique du RNL était pourtant l'étude de Goodman concernant les appendicites aiguës devant une douleur abdominale. La valeur du RNL était supérieure à celle du 
nombre absolu de polynucléaires neutrophiles classiquement utilisé. D’autres travaux concernant notamment les méningites en 2016 par l'équipe de Mentis, les pneumopathies communautaires par Yoon et al, montraient, en arguant le même rationnel que pour les bactériémies, que le RNL constituait un marqueur indépendant de cause bactérienne d'une méningite ou d'une affection pulmonaire ${ }^{(18,30)}$. Ces deux études étaient réalisées de façon rétrospective. Les infections bactériennes étaient prouvées par des critères microbiologiques dans le LCR ou des prélèvements respiratoires.

\section{Population étudiée :}

Notre objectif en réalisant cette étude était d'évaluer la valeur diagnostique du RNL dans une population de patients non sélectionnée, d'un secteur conventionnel d'hospitalisation avec les moyens utilisés en routine, afin de se rapprocher de la « vraie vie ». Les patients hospitalisés dans le service de médecine interne d'Amiens sont des patients de tout âge aux diagnostics variés, admis pour des pathologies inflammatoires, thrombotiques, néoplasiques et infectieuses. Les contraintes liées à ces patients et aux techniques ne permettent pas de documenter systématiquement l'infection et confirment le plus souvent le diagnostic après 24 à $48 \mathrm{~h}$ d'hospitalisation. Nous avons choisi d'exclure les patients traités par corticothérapie, immunosuppresseurs ou connus pour des pathologies hématologiques ou néoplasiques. Les traitements et pathologies de ces patients pouvaient modifier le nombre de lymphocytes et de polynucléaires neutrophiles, et gêner l'interprétation de nos résultats. Le nombre de ces patients était de 171 sur les 510 patients hospitalisés pour fièvre et /ou syndrome inflammatoire. Leur exclusion permet également de se rapprocher d'une population « vie réelle », la proportion de patients sous biothérapie et/ou corticothérapie étant plus fréquente chez les patients de médecine interne par rapport à un secteur conventionnel d'hospitalisation « tout venant ». La prise en compte de ces facteurs confondants dans une analyse stratifiée permettrait de savoir si le RNL pourrait être utilisé chez ces différentes catégories de patients, et avec quel seuil.

En restreignant les infections bactériennes à celles documentées, le risque aurait été d'exclure à tort des infections bactériennes avérées, diagnostiquées sur des critères pratiques utilisés quotidiennement par les cliniciens dans ces services hospitaliers. Pour diminuer l'incertitude diagnostique liée notamment au recueil rétrospectif, les diagnostics « indéfinis » $(\mathrm{N}=70)$ étaient exclus de l'analyse en per-protocole mais ceci aboutissait à une diminution de puissance en diminuant le nombre de sujets concernés. L'analyse de sensibilité réalisée en incluant ces patients permettait néanmoins d'obtenir des performances diagnostiques similaires du RNL pour le diagnostic d'infection bactérienne.

Les phénomènes d'immuno-senescence pourraient altérer l'interprétation de ce rapport par la 
diminution physiologique du nombre de lymphocytes alors que le nombre de polynucléaires neutrophiles reste identique. Sur la population de patients âgés de plus de 75 ans, la valeur du RNL était également significativement plus élevée dans les infections bactériennes, sans montrer de supériorité par rapport à la CRP. En revanche, pour obtenir une sensibilité et spécificité acceptables, le seuil de RNL était plus élevé, à 15. Le manque de données ne permettait pas de comparer ce rapport à la PCT. A notre connaissance, c'est la première fois que le RNL est étudié dans ce type de population.

\section{Le RNL :}

\section{Intérêt pratique au quotidien :}

Notre étude montre que dans cette population de patients hospitaliers « tout venant », le RNL est significativement plus élevé dans les infections bactériennes par rapport aux autres causes de fièvre et/ou syndrome inflammatoire. Il peut ainsi constituer une aide diagnostique à l'admission de ces patients. Son utilisation en routine en fait un outil facile, accessible au quotidien. La numération formule sanguine est en effet l'examen le plus prescrit en France avec des indications extrêmement variées ${ }^{(31)}$. Cette utilisation en fait un outil supplémentaire à l'identification rapide d'une cause bactérienne à une fièvre et/ou un syndrome inflammatoire, sans recourir à un nouveau prélèvement, à un nouveau marqueur. Notre étude permet d'interpréter la numération formule sanguine de façon plus optimale, en apportant un seuil plus précis pour un profil de patient fréquemment rencontré par un clinicien.

\section{Modalités d'utilisation du RNL}

Ce rapport reflète la réponse inflammatoire à un stress aigu, le plus souvent bactérien. On pouvait donc supposer que le seuil de 10 proposé dans les études précédentes était trop élevé dans le cas des infections localisées, la réponse cytokinique étant plus modérée. Gürol, dans son étude propose un seuil à 5 pour les infections bactériennes localisées. Néanmoins, cette définition était basée sur des taux de PCT qui ont montré leur insuffisance à mettre en évidence les infections localisées ${ }^{(15)}$. Mendis montrait des valeurs de sensibilité et spécificité optimales avec un RNL à 8 pour le diagnostic de méningite bactérienne et Yoon un RNL à 7 pour le diagnostic de pneumopathie bactérienne. Le test de Youden trouvait chez nos patients un seuil optimal à 8,2 pour le diagnostic d'infection bactérienne 
tous sites confondus, localisée ou systémique. Chez les patients de plus de 75 ans, ce rapport semblait montrer son efficacité diagnostique pour un seuil à 15 . Une étude à plus grande échelle serait souhaitable pour préciser ce seuil, le nombre de patients âgés de plus de 75 ans étant réduit dans notre population de patients.

\section{Intérêt pronostique?}

L'élévation du RNL est le reflet d'une réponse cytokinique intense, révélée par une majoration d'apoptose des lymphocytes et une élévation du nombre de polynucléaires neutrophiles circulants en réaction à une infection bactérienne et s'exprime donc de façon plus marquée dans les états septiques sévères, expliquant les valeurs plus élevées chez les patients bactériémiques et de façon beaucoup moins importante dans les infections localisées. Les principales études diagnostiques concernant le RNL étudiaient en effet son utilité pour le diagnostic de bactériémie ${ }^{(12,13,14,15,17)}$. Loonen dans son étude testait ce marqueur chez des patients suspects d'infection bactérienne alors qu'ils présentaient des critères de SRIS, révélant des valeurs de sensibilité supérieures à celles de la PCT et la CRP, pour un ratio supérieur à 10. La spécificité était inférieure à celle de la PCT quand celle-ci était supérieure à $2 \mathrm{ng} / \mathrm{mL}$. Les valeurs optimales de sensibilité et de spécificité étaient obtenues par un score composite associant une PCT $>2 \mathrm{ng} / \mathrm{mL}$ et un RNL $>12^{(12)}$. Les valeurs de RNL calculées chez nos patients ne permettaient pas d'identifier les patients bactériémiques ou en état septique sévère. Notre étude ne permettait de mettre en évidence que 23 bactériémies, soit $18 \%$ seulement de toutes les infections bactériennes. Notre population concernait des patients hospitalisés en secteur conventionnel. Les cas sévères étaient donc plus rarement admis dans ce service à leur sortie des urgences, ce qui peut expliquer le faible nombre de bactériémies et de patients sévères, limitant l'interprétation des données concernant ce diagnostic et le pronostic de ces patients. De plus, le recueil étant effectué de façon rétrospective, il est possible que certains patients bactériémiques n'aient pas bénéficié d'hémocultures pendant leur hospitalisation. Les données manquaient pour analyser la valeur du score composite PCT et RNL, la PCT n'étant pas dosée systématiquement à l'admission du patient.

Le nombre de patients entrant dans le critère composite de sévérité était ainsi faible. En effet, les études citées précédemment recueillaient ces données à l'admission aux urgences pour les pneumopathies ${ }^{(23)}$, chez tous les patients (en hospitalisation conventionnelle ou en soins intensifs)

pour les endocardites et les bactériémies ${ }^{(21,22,24)}$. Ce rapport est en effet le reflet des dommages tissulaires en rapport à une infection bactérienne et peut ainsi en révéler la sévérité et le risque de séquelles. De plus, le recueil des données à l'admission se réalisant de façon rétrospective, certaines 
données, notamment concernant la sévérité de la pathologie à l'admission, pouvaient manquer (fréquence respiratoire, état de vigilance...).

\section{Autres utilisations du RNL :}

Le RNL a souvent été montré comme un marqueur de l'inflammation, qu'elle soit chronique (en phase pré-thérapeutique comme marqueur pronostique dans les cancers ou pour le risque cardiovasculaire) ou aiguë dans le cadre des infections bactériennes. Son élévation correspond en effet à l'intensité des dommages tissulaires secondaires à des infections, des néoplasies ou en postopératoire. Le RNL des patients avec un diagnostic de cancer n'était pas significativement plus élevé comparé aux autres patients. Le recueil de données était cependant limité aux patients présentant une fièvre et/ou un syndrome inflammatoire et l'interprétation de ces données dans ce cadre doit être prudente. 


\section{CONCLUSION :}

Le RNL constitue donc une nouvelle approche de la numération formule sanguine pour argumenter le diagnostic d'infection bactérienne dans une population hospitalière de patients pour laquelle la documentation microbiologique n'est pas toujours possible. Notre étude ne permet pas de conclure sur l'interprétation de ces données concernant le diagnostic de bactériémie, ni sur le pronostic des patients. Les biais décrits précédemment pourraient être corrigés en établissant un recueil prospectif des données chez chaque patient hospitalisé en médecine interne pour syndrome inflammatoire et/ou hyperthermie. La définition d'infection bactérienne serait ainsi établie de façon plus précise, en s'astreignant à l'obtention des critères validés d'infection microbiologiques (interrogatoire ciblé, examen clinique précis et documentation microbiologique plus systématique). Une analyse en sousgroupes pourrait évaluer son utilisation en cas de traitement par corticoïdes ou immuno-suppresseurs ou en cas de cancer connu.

Notre équipe travaille actuellement de façon prospective sur les données concernant tous les patients présentant une fièvre et/ou un syndrome inflammatoire à l'admission dans le service de médecine interne du CHU d'Amiens (CRP, PCT, nombre de polynucléaires neutrophiles, RNL). L'analyse de ces données permettra de valider nos résultats concernant l'intérêt du RNL dans notre pratique clinique comme aide diagnostique à l'admission du patient en travaillant à corriger les différents biais décrits. 


\section{BIBLIOGRAPHIE}

(1) Riedel S. Procalcitonin and the role of biomarkers in the diagnosis and management of sepsis. Diagn Microbiol Infect Dis. 2012 Jul;73(3):221-7.

(2) Ropars A. Rôle physiologique et signification physiopathologique de la protéine C-Réactive (PCR). 2009. INSERM U961 Paris Institut Pasteur. Mise à jour le 15 décembre 2009. Disponible sur :www.comites- d- interface.inserm.fr/cint/content/download/20337/4082 5/version/1/file/ROPARS_15-12-09.pdf.

(3) Limper M, de Kruif MD, Duits AJ, Brandjes DP, van Gorp EC. The diagnostic role of Procalcitonin and other biomarkers in discriminating infectious from non-infectious fever. $\mathbf{J}$ Infect. 2010 Jun;60(6):409-16.

(4) Simon L, Gauvin F, Amre DK, Saint Louis P, Lacroix J. Serum Procalcitonin and C-Reactive Protein Levels as Markers of Bacterial Infection: A Systematic Review and Meta-analysis. Clin Infect Dis. 2004 Jul;39(2):206-17.

(5) Assicot M, Gendrel D, Carsin H, Raymond J, Guilbaud J, Bohuon C. High serum procalcitonin concentrations in patients with sepsis and infection. Lancet. 1993 Feb;341 (8844):515-8.

(6) Van der Does Y, Limper M,Schuit SC, Poley MJ, van Rosmalen J, Ramakers C, Patka P, van Gorp EC, Rood PP et al. Higher diagnostic accuracy and cost-effectiveness using procalcitonin in the treatment of emergency medicine patients with fever (The HiTEMP study) : a multicenter randomized study. BMC Emerg Med. 2016 Apr;(6)16:17.

(7) Wacker C, Prkno A, Brunkhorst FM, Schlattmann P. Procalcitonin as a diagnostic marker for sepsis: a systematic review and meta-analysis. Lancet Infect Dis. 2013 May;13(5):426-35.

(8) Butcher SK, Chahal H, Nayak L,Sinclair A, Henriquez NV, Sapey E et al. Senescence in innate immune responses: reduced neutrophil phagocytic capacity and CD16 expression in elderly humans. J Leukoc Biol. 2001 Dec;70(6):881-6.

(9) Crétel E, Veen I, Pierres A, Bongrand P, Gavazzi G. Immunosenescence and infections, myth or reality? Med Mal Infect. 2010 Jun;40(6):307-18.

(10) Van der Does Y, Rood PP, Haaqsma JA, Patka P, van Gorp EC, Limper M. Procalcitoninguided therapy for the initiation of antibiotics in the ED: a systematic review. Am J Emerg Med. 2016 Jul;34(7):1286-93.

(11) Zahorec R. Ratio of neutrophil to lymphocyte counts--rapid and simple parameter of systemic inflammation and stress in critically ill. Bratisl Lek Listy. 2001;102(1):5-14. 
(12) De Jager C, van Wijk PR, Mathoera RB, de Jongh-Leuvenink J, van der Poll T, Wever PC. Lymphocytopenia and neutrophil-lymphocyte count ratio predict bacteremia better than conventional infection markers in an emergency care unit. Crit Care. 2010;14(5):192.

(13) Loonen AJ, de Jager CP, Tosserams J, Kusters R, Hilbink M, Wever PC et al. Biomarkers and Molecular Analysis to Improve Bloodstream Infection Diagnostics in an Emergency Care Unit. PLoS One. 2014 Jan;9(1):87315.

(14) Lowsby R, Gomes C, Jarman I, Lisboa P, Nee PA, Vardhan M et al. Neutrophil to lymphocyte count ratio as an early indicator of blood stream infection in the emergency departement. Emerg Med J. 2015 Jul;32(7):531-4.

(15) Gürol G, Çiftci IH, Terizi HA, Atasoy AR, Ozbek A, Köroğlu M. Are there standardized cutoff values for neutrophil-lymphocyte ratios in bacteremia or sepsis? J Microbiology Biotechnol. 2015 Apr;25(4):521-5.

(16) Laukemann S, Kasper N, Kulkarni P, Steiner D, Rast AC, Kust A et al. Can We Reduce Negative Blood Cultures with Clinical Scores and Blood Markers? Results From an Observational Cohort Study. Medicine (Baltimore). 2015 Dec;94(49):2264.

(17) Shapiro NI, Wolfe RE, Wright SB, Moore R, Bates DW. Who needs a blood culture? A prospectively derived and validated prediction rule. J Emerg Med. 2008 Oct;35(3):255-264.

(18) Mentis AF, Kyprianou MA, Xirogianni A, Kesanopoulos K, Tzanakaki G. Neutrophil-tolymphocyte ratio in the differential diagnosis of acute bacterial meningitidis. Eur $\mathrm{J}$ Clin Microbiol Infect Dis. 2016 Mar;35(3):397-403.

(19) Tanriverdi H, Örnek T, Erboy F, Altinsoy B, Uyqur F, Atalay F et al. Comparison of diagnostic values of procalcitonin, C-reactive protein and blood neutrophil/lymphocyte ratio levels in predicting bacterial infection in hospitalized patients with acute exacerbations of COPD. Wien Klin Wochenschr. 2015 Oct;127(19-20):756-63.

(20) Bekdas M, Goksugur SB, Sarac EG, Erkocoglu M, Demircioglu F. Neutrophil/Lymphocyte and C-reactive protein/mean platelet volume ratios in differentiating between viral and bacterial pneumonias and diagnosing early complications in children. Saudi Med J. 2014 May;35(5):442-7.

(21) Turak O, Özcan F, Işleyen A, Başar FN, Gül M, Yilmaz S et al. Usefulness of neutrophil-tolymphocyte ratio to predict in-hospital outcomes in infective endocarditis. Can J Cardiol. 2013 Dec;29(12):1672-8.

(22) Bozbay M, Ugur M, Uyarel H, Cicek G, Koroglu B, Tusun E et al. Neutrophil-to-lymphocyte ratio as a prognostic marker in infective endocarditis: in hospital and long-term clinical results. J Heart Valve Dis. 2014 Sep;23(5):617-23. 
(23) De Jäger CP, Wever PC, Gemen EF, Kusters R, van Gageldonk-Lafeber AB, van der Poll T et al. The Neutrophil-Lymphocyte Count Ratio in Patients with Community-Acquired Pneumonia. Plos One. 2012; 7(10):46561.

(24) Riché F, Gayat E, Barthélémy R, Le Dorze M, Matéo J, Payen D. Reversal of neutrophil-tolymphocyte count ratio in early versus late death from septik shock. Crit Care. 2015 Dec;19:439.

(25) Raffi F, Cambau E, Garré M, Gaudelus J, Guery B, Guillot M et al. 17ème conférence de consensus en thérapeutique anti infectieuse. Prise en charge des méningites bactériennes aigües communautaires (à l'exclusion du nouveau-né). Méd et Mal Inf. 2008 Nov;39(3):145210.

(26) Mouton T, Birgé J, Bru JP, Geffray L, Massip P, Megarbane B et al. Prise en charge des infections des voies respiratoires basses de l'adulte immunocompétent. Méd et Mal Inf. 2006 Mar;36:235-244.

(27) Habib G, Hoen B, Tornos P, Thuny F, Prendergast B, Vilacosta I et al. ESC Guidelines for the management of infective endocarditis. The task force for the management of infective endocarditis of the European Society of Cardiology (ESC). Eur Heart J. 2015 Aug;30(9):2369-2413.

(28) Caron F, Galperine T, Etienne M, Merens A, Flateau C, Azria E et al. Diagnostic et antibiothérapie des infections urinaires bactériennes communautaires de l'adulte. Mise au point. Texte court. SPILF- Société de Pathologie Infectieuse de Langue Française 2015. Mise à jour décembre 2015. Disponible sur http://infectiologie.com

(29) Menthonnex E, Egard F, Torres JP. L'indice de gravité simplifié ambulatoire à la phase préhospitalière. La Revue des SAMU. 1997 ;1:59-64.

(30) Yoon N, Son C, Um SJ. Role of the Neutrophil-Lymphocyte Count Ratio in the differential diagnosis between pulmonary tuberculosis and bacterial community-acquired pneumonia. Ann Lab Med. 2013 Mar;33(2):105-10.

(31) Troussard X, Vol S, Cornet E, Bardet V, Couaillac JP, Fossat C. et al. Etude des valeurs normales de l'hémogramme chez l'adulte: un besoin pour une meilleure interprétation et pour 1'accréditation du laboratoire. Annales de Biologie Clinique. 2014 ;72 (5) :561-581. 